\title{
Multi-Level Genetic Algorithm (GA)-based Acoustic-Elastodynamic Imaging of Coupled Fluid-Solid Media to Detect an Underground Cavity
}

\author{
Bruno P. Guidio ${ }^{1}$, Paula Ribeiro², Boo Hyun $\mathrm{Nam}^{3}$, and Chanseok Jeong ${ }^{4,}$ \\ ${ }^{1}$ Postdoctoral Researcher, School of Engineering and Technology, Central Michigan \\ University, Mount Pleasant, MI 48859, USA; ORCID: 0000-0003-1943-8806 \\ ${ }^{2}$ Federal University of Bahia, Salvador, BA 40170, Brazil \\ ${ }^{3}$ Associate Professor, Department of Civil, Environmental, and Construction Engineering, \\ University of Central Florida, Orlando, FL 32816, USA \\ ${ }^{4}$ Assistant Professor, School of Engineering and Technology, Central Michigan University, \\ Mount Pleasant, MI 48859, USA; ORCID: 0000-0002-0488-8559 \\ *Corresponding author: jeong1c@cmich.edu
}

\begin{abstract}
This work studies the feasibility of imaging a coupled fluid-solid system by using the elastodynamic and acoustic waves initiated from the top surface of a computational domain. We consider a onedimensional system, where a fluid layer is surrounded by two solid layers. The bottom solid layer is truncated by using a wave-absorbing boundary condition (WABC). We measure the wave responses on a sensor located on the top surface, and the measured signal contains information about the underlying physical system. By using the measured wave responses, we identify the elastic moduli of the solid layers and the depths of the interfaces between the solid and fluid layers. We employ a multi-level Genetic Algorithm (GA) combined with a frequency-continuation scheme to invert for the values of sought-for parameters. The numerical results show the following findings. First, the depths of solid-fluid interfaces and elastic moduli can be reconstructed by the presented method. Second, the frequency-continuation scheme improves the convergence of the estimated values of parameters toward their targeted values. Lastly, a preliminary inversion, using an all-solid model, can be employed to identify if a fluid layer is presented in the model by showing one layer with a very large value of Young's modulus (with a similar value to that of the bulk modulus of water) and the value of mass density being similar to that of water. Then, the primary GA inversion method, based on a fluid-solid model, can be utilized to adjust the soil characteristics and fine-tune the locations of the fluid layer. If this work is extended to a 3D setting, it can be instrumental to finding unknown locations of fluid-filled voids in geological formations that can lead to ground instability and/or collapse (e.g., natural/anthropogenic sinkhole, urban cave-in subsidence, etc.).
\end{abstract}

\section{Introduction}

Detecting underground cavities (e.g., karstic cavities, caves, tunnels, etc.) is a challenging task for geotechnical engineering projects due to the geological/hydrogeological complexity of the subsurface environment. Geological hazards, such as collapsing soil or urban ground collapse due to subsurface voids, could induce significant damage to infrastructures. Such hazard is one of the major issues for land planning, infrastructure operation and maintenance, and disaster management. Cavity evolution that occurs due to hydrogeological process may cause sinkhole collapse. For example, Florida's karst environment involves active groundwater recharge to the Floridan Aquifer that makes overburden soils to be eroded; thus, as shown in Fig. 1, a cavity could gradually grow, leading to a sudden collapse (cover-collapse type) or gradual subsidence (cover-subsidence type) (Beck, 1986; Tihansky, 1999; Xiao et al., 2016; Kim et al., 2020; Nam et al., 2020). These large underground water-filled cavities hidden below building and transportation infrastructures should be pre-detected so that prevention and mitigation measures are applied before catastrophic collapse or excessive ground settlement takes place.

Cone penetration test (CPT) and standard penetration test (SPT) have been employed for detecting and 

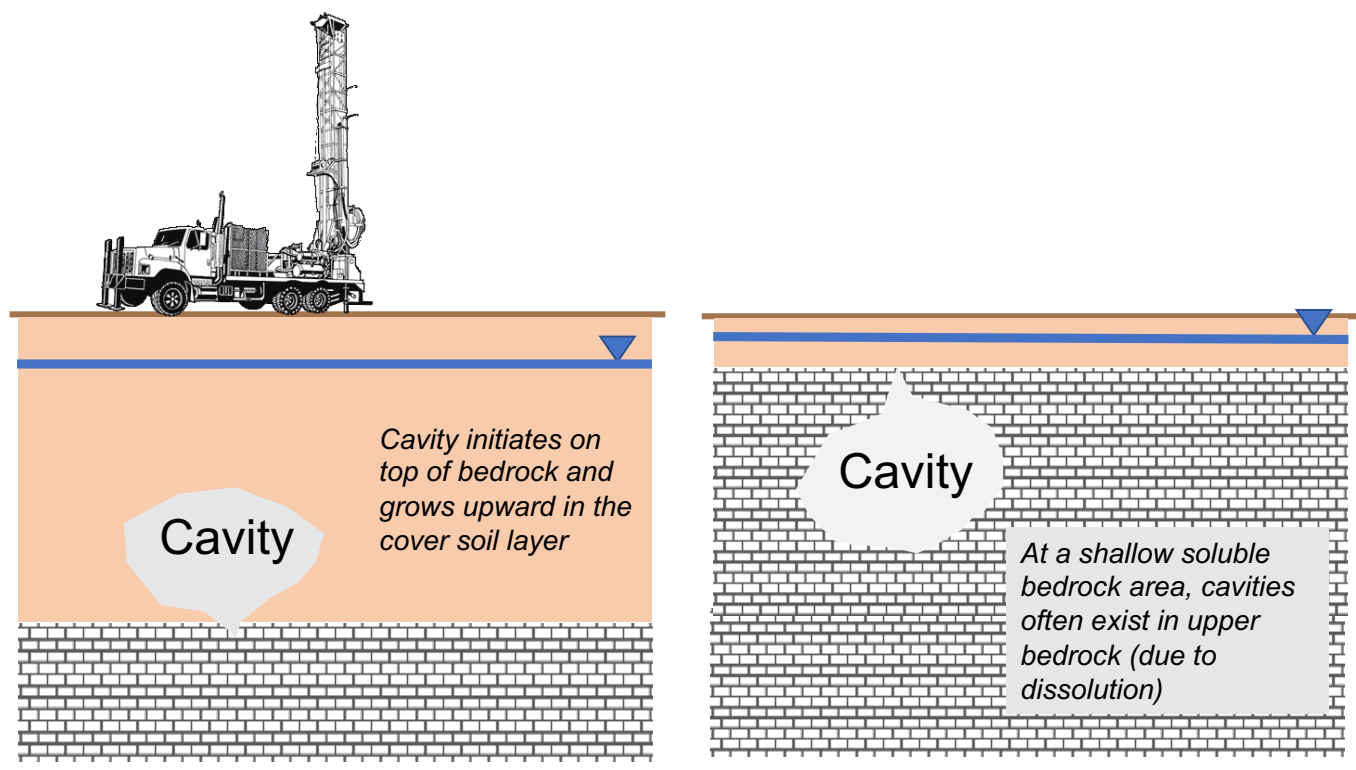

Figure 1: Schematic diagram of water-filled subsurface cavity (common in Florida's karst environment): (a) cavity in either cover soils layer and (b) cavity within near surface of limestone bedrock.

characterizing subsurface cavities in a cover soil layer, referred to as a raveled zone in the karst areas (Bloomberg et al., 1988; Shamet et al., 2017; Nam et al., 2018; Nam and Shamet, 2020). Although SPT and CPT provide a continuous subsurface profile - revealing soil type, resistance, and stratigraphy-, these invasive methods only collect point-wise profiles of the subsurface environment. As such, it is time-consuming and labor-intensive to apply CPT and SPT to wide areas multiple times.

Numerous geophysical studies over the past decades have introduced nondestructive evaluation (NDE) methods for detecting and estimating the geometry of subsurface cavities. For example, ground-penetrating radar (GPR), micro-gravimetry, and resistivity imaging and their combinations can characterize an underground cavity based on its size and depth position (Cardarelli et al., 2003; Mochales et al., 2008; Fehdi et al., 2014; Kiflu et al., 2016). Namely, GPR is inefficient in highly heterogeneous media-such as backfills, moist clays with high soil conductivity, and saturated soils below the groundwater table. Micro-gravimetric method is effective in detecting shallow cavities (Butler, 1984; Bishop et al., 1997), but its density contrast cannot be obtained clearly if the size of a void is relatively small compared to its depth. An electrical resistivity method has been applied to detect air-filled or water-filled cavities (Van Schoor, 2002; Coşkun, 2012), but it requires a large areal space for surveying and cannot detect small-scale irregularities in the geologic interfaces since it measures averaged resistivity values and is easily ruined by noise sources, such as piping, power lines, and house structures.

On the other hand, elastodynamic imaging has been widely used in site characterization (Roesset et al., 1990; Brown et al., 2002; Kallivokas et al., 2013). It hinges on elastodynamic waves that are initiated by wave sources on the ground surface and/or borehole sources, and, then, are reflected/refracted due to material heterogeneity within a medium. The resulting wave motions can be measured on the ground surface or at borehole seismic arrays. The inverse modeling, associated with elastic wave propagation analyses, provides promising results of identifying the material properties of the media. Inverse modelingemploying a partial differential equation (PDE)-constrained optimization method and the finite or spectral element method (FEM or SEM) - has been utilized to infer the spatial distribution of material properties of host media in fine resolution (Kang and Kallivokas, 2011; Fathi, 2015; Fathi et al., 2015a). Specifically, for instances, the geotechnical site characterization methodologies have been investigated in a soil domain that is truncated by Perfectly-Matched-Layers (PML), where waves decay and are not reflected off surrounding boundaries (Kang and Kallivokas, 2010b; Kucukcoban and Kallivokas, 2011, 2013; Fathi et al., 2015b; Mashayekh et al., 2018; Poul and Zerva, 2018), by using state-adjoint-control equation-based fullwaveform inversion (FWI) approaches (Kallivokas et al., 2013; Kang and Kallivokas, 2010a, 2011; Fathi et al., 2015a, 2016; Pakravan et al., 2016; Kucukcoban et al., 2019). The Gauss-Newton-based FWI method, which is based on a gradient vector and a Hessian matrix, had been studied for characterizing the material profiles in a truncated two-dimensional solid domain (Tran and McVay, 2012; Pakravan et al., 2016) for the appli- 
cation of the site characterization. The PDE-constrained optimization has been also used in consideration of the boundary element method (BEM), of which computational efficiency is much greater than the FEM due to the reduction of the dimensionality, to detect the geometries of wave-scattering objects in host media. Namely, there have been studies on inverse scattering algorithms using BEM wave solvers, hinging on the moving boundary concept and the total derivative (Petryk and Mroz, 1986) that allows for computing the derivative of an objective functional with respect to the geometry variables of scattering objects (Guzina et al., 2003; Jeong and Kallivokas, 2008; Jeong et al., 2009). The extended finite element method (XFEM) has been used as a wave solver of the studies to identify cracks or air-filled voids in solid media because it allows for avoiding expensive remeshing process in inverse iterations while using the flexibility of the FEM for heterogeneous materials (Jung et al., 2013; Jung and Taciroglu, 2014, 2016). In addition, a frequency-continuation scheme was devised to help the inversion solver tackle the multiplicity of solutions of subsurface imaging problems (Bunks et al., 1995; Na and Kallivokas, 2008; Kang and Kallivokas, 2010a; Fathi et al., 2015a). That is, when the convergence rate of the inversion solver is decreased due to a local minimum of an objective functional that is comprised of measurement data corresponding to a given dominant frequency of excitation, another set of measurement data that are induced by excitation of a different dominant frequency are employed. Such a frequency shift can change the curvature of the objective functional so that a minimizer can escape a local minimum. In general, one may consider increasing frequency from one inversion set to another because it has been reported that using a lower frequency leads to the inversion result of a lower resolution (due to a larger wavelength), then, one can fine-tune it using a higher frequency (due to a smaller wavelength) ( $\mathrm{Na}$ and Kallivokas, 2008).

Despite such recent developments in elastodynamic wave imaging techniques, few papers have demonstrated the feasibility of identifying the properties of a solid system that includes fluid-filled voids. There have been a finite difference method (FDM)-based FWI approaches, which employed all solid elements in an entire computational domain and detect the areas with smaller values of shear wave speeds $V_{s}$ (e.g., around $100 \mathrm{~m} / \mathrm{s}$ ) than those of typical soils and rocks to indicate air-filled voids (Tran and McVay, 2012; Tran et al., 2013; Mirzanejad et al., 2020). However, those works have not explicitly modeled the interfaces between air voids and the solid domain. Thus, the authors (henceforth, we) are concerned that, first, modeling a fluid domain as solid elements with a small value of $V_{s}$ could introduce numerical error in forward wave solutions. Second, using a small value of $V_{s}$ requires a small size of a solid element for wave simulations. However, the aforementioned FDM works did not use such a small-sized element to model a void so that it could suffer from the additional error of wave solutions. To address this issue, we suggest to explicitly use fluid elements to model voids filled with water (or air) so that accurate fluid-solid coupling (Lloyd et al., 2016) should be incorporated into the wave solver. Then, by using such a wave solver, inverse modeling could accurately identify the interfaces between fluid and solids as well as the material profiles of solids. As a prototype work of our suggested method, this paper investigates the feasibility to estimate the interfaces between fluid and solid layers and the material properties of solid layers in a multi-layered system by using acoustic and elastodynamic waves generated from the ground surface. Such investigation will prove the feasibility of detecting a water-filled void and identifying its location and geometry in soils, to which overlaying ground surface could potentially sink.

This work uses the FEM to study the wave responses of the coupled fluid-solid media in a prototype onedimensional setting. The solid formation of a semi-infinite extent is truncated by using an absorbing boundary, and a proper fluid-solid interface condition is considered. The inverse problem is cast into a minimization problem, where the value of an error between the synthetic measured wave response due to target profiles and the computed counterpart due to guessed profiles is minimized. The solution multiplicity of the minimization process is addressed by using the multi-level Genetic Algorithm (GA) in this work. Under this new scheme, we suggest the following steps: (i) The optimizer identifies an inversion solution around a local minimum after a number of GA iterations in the first-level GA; (ii) Then, we can introduce a misfit function for a different measurement signal induced by a pulse signal of a different central frequency in the next-level GA; ( iii) By doing so, the GA optimizer is able to escape the local minimum of the previouslevel GA; $(i v)$ The same technique is employed to address the solution multiplicity of the next-level GA. The numerical results show that our numerical simulations, using the multiple-frequency-level GA, accurately detect the interfaces between fluid and solid layers and the material properties of solid layers. We also demonstrate that a preliminary GA inversion can tell whether the subsurface media include a fluid layer or not. Namely, the preliminary inversion can indicate the existence of a fluid layer by detecting a very large value of Young's modulus (with a similar value to that of the bulk modulus of water) and a mass density with a similar value to that of water. Once the existence of a fluid layer is detected, a primary GA inversion, using the fluid-solid model, can further estimate the locations of a fluid layer and the material properties 
of surrounding solids.

\section{Problem Definition}

We aim to identify the depths of the interfaces between a fluid layer and its surrounding solid layers and the stiffness of the solid layers by using a dynamic test, which generates elastodynamic waves into the subsurface media from the top surface.

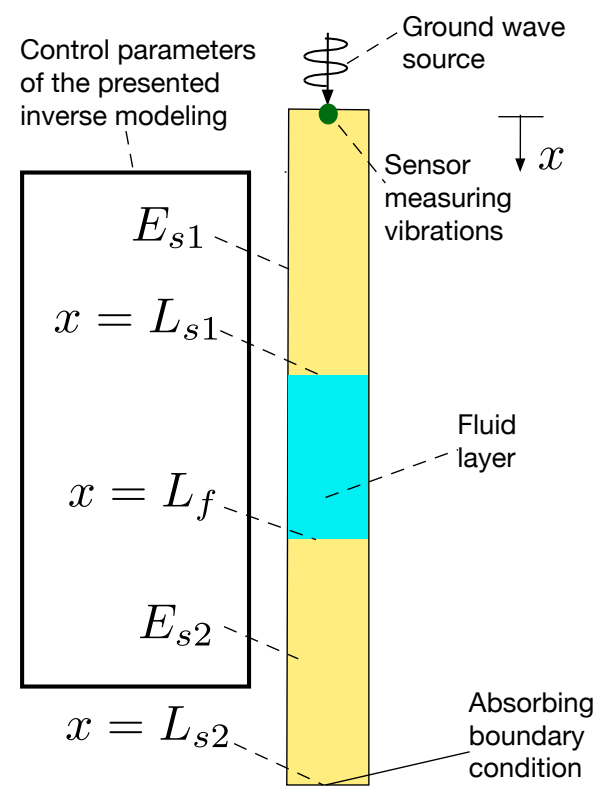

Figure 2: Problem schematic.

We consider a 1D fluid-solid model, which consists of three different layers, i.e., two solid layers and one fluid layer (see Fig. 2). The governing differential equations for the compressional waves in the three layers are given by:

$$
\begin{array}{rlrl}
\frac{\partial}{\partial x}\left(E_{s 1} \frac{\partial u_{s 1}}{\partial x}\right) & =\rho_{s 1} \frac{\partial^{2} u_{s 1}}{\partial t^{2}}, & & 0 \leq x \leq L_{s 1}, \\
\frac{\partial^{2} P}{\partial x^{2}} & =\frac{1}{c_{f}^{2}} \frac{\partial^{2} P}{\partial t^{2}}, & L_{s 1} \leq x \leq L_{f}, \\
\frac{\partial}{\partial x}\left(E_{s 2} \frac{\partial u_{s 2}}{\partial x}\right) & =\rho_{s 2} \frac{\partial^{2} u_{s 2}}{\partial t^{2}}, & & L_{f} \leq x \leq L_{s 2},
\end{array}
$$

where $L_{s 1}, L_{f}$, and $L_{s 2}$ denote, respectively, the depth of the interface between the top solid layer and the fluid layer, that between the fluid and the bottom solid layer, and the depth of the truncation wave absorbing boundary. Also, $u_{s 1}(x, t)$ and $u_{s 2}(x, t)$ denote the displacement fields of the wave motions of solid particles in the top and bottom solid layers. $P$ denotes the fluid pressure field of the wave motions in the fluid. The wave speeds of the three layers are given by $c_{s 1}=\sqrt{\frac{E_{s 1}}{\rho_{s 1}}}, c_{f}=\sqrt{\frac{\kappa_{f}}{\rho_{f}}}$, and $c_{s 2}=\sqrt{\frac{E_{s 2}}{\rho_{s 2}}}$, respectively, where $E, \rho$, and $\kappa$ are the modulus of elasticity, the density, and the bulk modulus. The problem of interest also includes the following boundary conditions:

$$
\begin{aligned}
E_{s 1} \frac{\partial u_{s 1}}{\partial x} & =-f(t), & & x=0, \\
\frac{\partial u_{s 2}}{\partial x} & =\frac{-1}{c_{s 2}} \frac{\partial u_{s 2}}{\partial t}, & & x=L_{s 2},
\end{aligned}
$$

A pulse signal $f(t)$ is applied at the top of the solid layer at $x=0$ (per (4)). The depth of the bottom solid layer is truncated by using the absorbing boundary condition (per (5)). The solid and fluid layers are 
coupled via the following interface conditions:

$$
\begin{aligned}
E_{s 1} \frac{\partial u_{s 1}}{\partial x} & =-P, & x & =L_{s 1}, \\
\frac{\partial P}{\partial x} & =-\rho_{f} \frac{\partial^{2} u_{s 1}}{\partial t^{2}}, & x & =L_{s 1}, \\
E_{s 2} \frac{\partial u_{s 2}}{\partial x} & =-P, & x & =L_{f}, \\
\frac{\partial P}{\partial x} & =-\rho_{f} \frac{\partial^{2} u_{s 2}}{\partial t^{2}}, & & x=L_{f} .
\end{aligned}
$$

The system is initially at rest:

$$
\begin{array}{lc}
u_{s 1}=u_{s 2}=P=0, & t=0, \\
\frac{\partial u_{s 1}}{\partial t}=\frac{\partial u_{s 2}}{\partial t}=\frac{\partial P}{\partial t}=0, & t=0 .
\end{array}
$$

\section{Finite element modeling to compute the wave responses.}

To derive the weak form, the governing wave equations in the strong form (1)-(3) are multiplied by the test functions $v_{1}(x), w(x)$, and $v_{2}(x)$. By integrating them by parts and using the boundary and interface conditions of the strong form, the following weak forms are obtained:

$$
\begin{aligned}
\int_{0}^{L_{s 1}}\left[E_{s 1} \frac{\partial v_{1}}{\partial x} \frac{\partial u_{s 1}}{\partial x}+\rho_{s 1} v \frac{\partial^{2} u_{s 1}}{\partial t^{2}}\right] d x & =v(0) f(t)-v\left(L_{s 1}\right) P\left(L_{s 1}\right), \\
\int_{L_{s 1}}^{L_{f}}\left[\kappa_{f} \frac{\partial w}{\partial x} \frac{\partial P}{\partial x}+\rho_{f} w \frac{\partial^{2} P}{\partial t^{2}}\right] d x & =\rho_{f} \kappa_{f}\left[w\left(L_{s 1}\right) \frac{\partial^{2} u_{s 1}}{\partial t^{2}}\left(L_{s 1}\right)-w\left(L_{f}\right) \frac{\partial^{2} u_{s 2}}{\partial t^{2}}\left(L_{f}\right)\right], \\
\int_{L_{f}}^{L_{s 2}}\left[E_{s 2} \frac{\partial v_{2}}{\partial x} \frac{\partial u_{s 2}}{\partial x}+\rho_{s 2} v_{2} \frac{\partial^{2} u_{s 2}}{\partial t^{2}}\right] d x & =v_{2}\left(L_{f}\right) P\left(L_{f}\right)-E_{s 2} \sqrt{\frac{\rho_{s 2}}{E_{s 2}}} v_{2}\left(L_{s 2}\right) \frac{\partial u_{s 2}}{\partial t}\left(L_{s 2}\right) .
\end{aligned}
$$

Then, we approximate the functions as:

$$
\begin{gathered}
v_{1}(x)=\mathbf{v}_{1}^{T} \boldsymbol{\phi}(x), \quad u_{s 1}(x, t)=\boldsymbol{\phi}(x)^{T} \mathbf{u}_{s 1}(t), \\
w(x)=\mathbf{w}^{T} \boldsymbol{\Psi}(x), \quad P(x, t)=\boldsymbol{\Psi}(x)^{T} \mathbf{P}(t), \\
v_{2}(x)=\mathbf{v}_{2}^{T} \boldsymbol{\Omega}(x), \quad u_{s 2}(x, t)=\boldsymbol{\Omega}(x)^{T} \mathbf{u}_{s 2}(t),
\end{gathered}
$$

where $\phi(x), \boldsymbol{\Psi}(x)$, and $\boldsymbol{\Omega}(x)$ denote vectors of global basis functions constructed by local shape functions, and $\mathbf{u}_{s 1}, \mathbf{P}$, and $\mathbf{u}_{s 2}$ are the vectors of nodal solutions. Introducing the finite element approximations (15)(17) into the weak forms (12)-(14) provides the following discrete form:

$$
\begin{aligned}
& \mathbf{K}_{s 1} \mathbf{u}_{s 1}+\mathbf{M}_{s 1} \frac{\partial^{2} \mathbf{u}_{s 1}}{\partial t^{2}}=\mathbf{f}-\mathbf{L}_{1} \mathbf{P}\left(L_{s 1}\right), \\
& \mathbf{K}_{f} \mathbf{P}+\mathbf{M}_{f} \frac{\partial^{2} \mathbf{P}}{\partial t^{2}}=\mathbf{L}_{2} \rho_{f} \kappa_{f} \frac{\partial^{2} \mathbf{u}_{s 1}}{\partial t^{2}}-\mathbf{L}_{3} \rho_{f} \kappa_{f} \frac{\partial^{2} \mathbf{u}_{s 2}}{\partial t^{2}}, \\
& \mathbf{K}_{s 2} \mathbf{u}_{s 2}+\mathbf{M}_{s 2} \frac{\partial^{2} \mathbf{u}_{s 2}}{\partial t^{2}}=-\sqrt{\rho_{s 2} E_{s 2}} \mathbf{L}_{5} \frac{\partial \mathbf{u}_{s 2}}{\partial t}+\mathbf{L}_{4} \mathbf{P},
\end{aligned}
$$


where the matrices are defined as:

$$
\begin{aligned}
\mathbf{K}_{s 1} & =\int_{0}^{L_{s 1}} E_{s 1} \frac{\partial \boldsymbol{\phi}(x)}{\partial x} \frac{\partial \boldsymbol{\phi}(x)^{T}}{\partial x} d x, \quad \mathbf{M}_{s 1}=\int_{0}^{L_{s 1}} \rho_{s 1} \phi(\boldsymbol{x}) \boldsymbol{\phi}(\boldsymbol{x})^{\boldsymbol{T}}, \\
\mathbf{K}_{f} & =\int_{L_{s 1}}^{L_{f}} \kappa_{f} \frac{\partial \boldsymbol{\Psi}(x)}{\partial x} \frac{\partial \boldsymbol{\Psi}(x)^{T}}{\partial x} d x, \quad \mathbf{M}_{f}=\int_{L_{s 1}}^{L_{f}} \rho_{f} \boldsymbol{\Psi}(\boldsymbol{x}) \boldsymbol{\Psi}(\boldsymbol{x})^{\boldsymbol{T}}, \\
\mathbf{K}_{s 2} & =\int_{L_{f}}^{L_{s 2}} E_{s 2} \frac{\partial \boldsymbol{\Omega}(x)}{\partial x} \frac{\partial \boldsymbol{\Omega}(x)^{T}}{\partial x} d x, \quad \mathbf{M}_{s 2}=\int_{L_{f}}^{L_{s 2}} \rho_{s 2} \boldsymbol{\Omega}(\boldsymbol{x}) \boldsymbol{\Omega}(\boldsymbol{x})^{\boldsymbol{T}} d x, \\
\mathbf{L}_{1} & =\boldsymbol{\phi}\left(L_{s 1}\right) \boldsymbol{\Psi}^{T}\left(L_{s 1}\right)=\left[\begin{array}{ccc}
0 & \cdots & 0 \\
\vdots & \ddots & \vdots \\
1 & \cdots & 0
\end{array}\right], \quad \mathbf{L}_{2}=\boldsymbol{\Psi}\left(L_{s 1}\right) \boldsymbol{\phi}^{T}\left(L_{s 1}\right)=\left[\begin{array}{ccc}
0 & \cdots & 1 \\
\vdots & \ddots & \vdots \\
0 & \cdots & 0
\end{array}\right], \\
\mathbf{L}_{3} & =\boldsymbol{\Psi}\left(L_{f}\right) \boldsymbol{\Omega}^{T}\left(L_{f}\right)=\left[\begin{array}{ccc}
0 & \cdots & 0 \\
\vdots & \ddots & \vdots \\
1 & \cdots & 0
\end{array}\right], \quad \mathbf{L}_{4}=\boldsymbol{\Omega}\left(L_{f}\right) \boldsymbol{\Psi}^{T}\left(L_{f}\right)=\left[\begin{array}{ccc}
0 & \cdots & 1 \\
\vdots & \ddots & \vdots \\
0 & \cdots & 0
\end{array}\right], \\
\mathbf{L}_{5} & =\boldsymbol{\Omega}\left(L_{s 2}\right) \boldsymbol{\Omega}^{T}\left(L_{s 2}\right)=\left[\begin{array}{ccc}
0 & \cdots & 0 \\
\vdots & \ddots & \vdots \\
0 & \cdots & 1
\end{array}\right],
\end{aligned}
$$

and the force vector is $\mathbf{f}=\phi(0) f(t)$. The discrete equations (18)-(20) lead to the following coupled discrete form:

$$
\begin{aligned}
\underbrace{\left[\begin{array}{ccc}
\mathbf{K}_{s 1} & \mathbf{L}_{1} & \mathbf{0} \\
\mathbf{0} & \mathbf{K}_{f} & \mathbf{0} \\
\mathbf{0} & -\mathbf{L}_{4} & \mathbf{K}_{s 2}
\end{array}\right]}_{\mathbf{K}}\left[\begin{array}{c}
\mathbf{u}_{s 1} \\
\mathbf{P} \\
\mathbf{u}_{s 2}
\end{array}\right] & +\underbrace{\left[\begin{array}{ccc}
\mathbf{0} & \mathbf{0} & \mathbf{0} \\
\mathbf{0} & \mathbf{0} & \mathbf{0} \\
\mathbf{0} & \mathbf{0} & E_{s 2} \sqrt{\frac{\rho_{s 2}}{E_{s 2}}} \mathbf{L}_{5}
\end{array}\right]}_{\mathbf{C}}\left[\begin{array}{c}
\frac{\partial \mathbf{u}_{s 1}}{\partial t} \\
\frac{\partial \mathbf{P}}{\partial t} \\
\frac{\partial \mathbf{u}_{s 2}}{\partial t}
\end{array}\right] \\
+ & \underbrace{\left[\begin{array}{ccc}
\mathbf{M}_{s 1} & \mathbf{0} & \mathbf{0} \\
-\rho_{f} \kappa_{f} \mathbf{L}_{2} & \mathbf{M}_{f} & \rho_{f} \kappa_{f} \mathbf{L}_{3} \\
\mathbf{0} & \mathbf{0} & \mathbf{M}_{s 2}
\end{array}\right]}_{\mathbf{M}}\left[\begin{array}{c}
\frac{\partial^{2} \mathbf{u}_{s 1}}{\partial t^{2}} \\
\frac{\partial^{2} \mathbf{P}}{\partial t^{2}} \\
\frac{\partial^{2} \mathbf{u}_{s 2}}{\partial t^{2}}
\end{array}\right]=\left[\begin{array}{l}
\mathbf{f} \\
\mathbf{0} \\
\mathbf{0}
\end{array}\right],
\end{aligned}
$$

which, at a discrete time $t_{n}$, can be written as:

$$
\mathbf{M} \ddot{\mathbf{q}}_{n}+\mathbf{C} \dot{\mathbf{q}}_{n}+\mathbf{K} \mathbf{q}_{n}=\mathbf{F}_{n},
$$

where $\mathbf{q}_{n}=\left[\mathbf{u}_{s 1}^{T}, \mathbf{P}^{T}, \mathbf{u}_{s 2}^{T}\right]^{T}$ is the vector containing the nodal solutions of solid displacements and acoustic pressures at the $n$-th time step, and $\dot{\mathbf{q}}_{n}$ and $\mathbf{q}_{n}$ denote the first and second-order derivatives of $\mathbf{q}_{n}$ with respect to time. We solve the second-order ordinary differential equation (28) by using the Newmark's unconditionally-stable time integration scheme (Newmark, 1959).

\section{Inverse Modeling}

We aim to identify the four control parameters $\left(E_{s 1}, E_{s 2}, L_{s 1}\right.$, and $\left.L_{f}\right)$ by using measured wave responses initiated by a known excitation signal $f(t)$. Here, the values of the other variables $\left(L_{s 2}, \rho_{s 1}, \rho_{s 2}, \kappa_{f}\right.$, and $\left.\rho_{f}\right)$ are set to be known during the inversion process, and we also know the excitation signal $f(t)$. We formulate this problem into a minimization problem to identify estimated control parameters that could lead to the minimum of an objective functional:

$$
\mathcal{L}=\int_{0}^{T}\left(u_{\mathrm{m}}(0, t)-u(0, t)\right)^{2} d t .
$$

In (29), $u_{\mathrm{m}}(0, t)$ denotes a wave signal recorded by the sensor at $x=0$ due to a target profile of the control parameters, and $u(0, t)$ denote computed counterpart at the same measurement location due to a guessed profile. In this computational study, $u_{\mathrm{m}}(0, t)$ is synthetically obtained by running the presented 
FEM solver using a target profile as an input. To prevent an inverse crime from taking place, we use smaller values of element sizes when $u_{\mathrm{m}}(0, t)$ is computed than when $u(0, t)$ is computed.

This work uses GA, which is a heuristic algorithm to reconstruct the control parameters that are the fittest to the objective of the problem. In each generation of GA, there are individuals, each of which has a set of different values of control parameters. In this work, GA runs the FEM wave solver, for the estimated values of control parameters of each individual, to compute $u(0, t)$ and evaluate the misfit (29). Then, GA evaluates the fitness of each individual by using the value of its misfit. Then, GA weeds out less-fitting individuals when it creates the next generation of individuals, each of which is characterized by its own set of estimated parameters. In this process, by virtue of the mutation process, where GA learns the better-fitting characteristics of control parameters, GA gives rise to new individuals in the next generation. The GA calculates the sensitivity of the fitness function with respect to the changes of the values of the control parameters and determines how a next generation of individuals is created. Namely, GA repeats the tasks of weeding out, selecting the best individual, and mutating at each generation. Therefore, our inverse modeling solver could identify targeted values of the parameters by using the iterative process of GA, where the values of the estimated parameters of the best-fit individual evolve over the progress of generations.

In the overall GA process under this problem, we impose constraints on the allowable ranges of each control parameter. For instance, we set $L_{s 1}$ (the depth of upper face of a fluid layer) is always smaller than $L_{f}$ (the depth of the lower face) such that the thickness of the fluid layer is always positive. We also set the values of elastic moduli $E_{s 1}$ and $E_{s 2}$ are always positive.

It is known that, in the minimization problem of a small number (e.g., less than 20) of control parameters, GA is likely to find a set of control parameters that are close to the global minimum (Jeong et al., 2017). Since there are only four control parameters, we originally hypothesized that the GA is a suitable solution approach to finding the global minimum solution of this inverse problem. However, the numerical experiments show that the GA suffers from the solution multiplicity, and, thus, we test a new multiple frequency-level GA approach. Namely, after the first level of GA is finished, our inversion solver uses the reconstructed values from the first level to define the upper and lower limits of the control parameters in the next GA level, which uses a pulse signal of a different central frequency from its predecessor, creating a new synthetic $u_{m}$. In the final-level GA, the final best-fit estimated control parameters are obtained as the inversion solution.

\section{Numerical Experiments}

This section shows a set of numerical experiments, studying the performance of the presented multilevel GA-based parameter-estimation method. In the first two examples, three targeted fluid-solid models, which differ from each other in terms of $L_{s 1}, L_{f}$, and $L_{s 2}$, are considered as follows. Model 1 has a total length $L_{s 2}$ of $60 \mathrm{~m}$ and its fluid is located between $x$ of $L_{s 1}=20 \mathrm{~m}$ and $x$ of $L_{f}=25 \mathrm{~m}$. The corresponding geometry information of Model 2 are $L_{s 2}$ of $60 \mathrm{~m}, L_{s 1}$ of $40 \mathrm{~m}$, and $L_{f}$ of $45 \mathrm{~m}$, and those of Model 3 are $L_{s 2}$ of $120 \mathrm{~m}, L_{s 1}$ of $50 \mathrm{~m}$, and $L_{f}$ of $80 \mathrm{~m}$. $L_{s 1}$ and $L_{f}$ are unknown parameters in the inversion. In all the models, the mass density $(\rho)$ of the solid layers is $2000 \mathrm{~kg} / \mathrm{m}^{3}$; their Young's moduli are $E_{s 1}=2 \times 10^{8}$ $\mathrm{Pa}$ and $E_{s 2}=5 \times 10^{8} \mathrm{~Pa}$; and the bulk modulus $\left(k_{f}\right)$ and mass density $\left(\rho_{f}\right)$ of the fluid layer are $2.34 \times 10^{9}$ $\mathrm{N} / \mathrm{m}^{2}$ and $1021 \mathrm{~kg} / \mathrm{m}^{3}$, respectively. While $E_{s 1}$ and $E_{s 2}$ are set to be unknown, $\rho, k_{f}$, and $\rho_{f}$ are set to be known in the inversion.

In the presented numerical experiments, a three-level GA-based inversion method is used with a frequencycontinuation scheme. Namely, in each GA set, a dynamic force with a different frequency content is used as follows.

- The upper and lower bounds of each control parameter are set for the first-level GA, and its lastgeneration leads to the best-fit individual.

- Then, in the second and third-level GA, the upper and lower limits of the four control parameters are updated with respect to the final-reconstructed values of the parameters obtained in the previouslevel GA. Namely, in the second-level GA, the values of the estimated $L_{s 1}$ and $L_{f}$ are bounded using the same upper and lower limits as the first GA level, while the values of the estimated $E_{s 1}$ and $E_{s 2}$ are bounded by using $\pm 50 \%$ derivations of their final-reconstructed values obtained during the first GA level.

- In the third GA level, the values of estimated $L_{s 1}$ and $L_{f}$ are bounded by using $\pm 5 \%$ derivations 
of their reconstructed values from the second GA level, while $\pm 10 \%$ derivations of the values of estimated $E_{s 1}$ and $E_{s 2}$ that are reconstructed from second-level GA level are used as their bounds in the last GA level.

- The number of generations (GN) and population size (PS) are both 50 in all three GA levels.

In order to avoid an inverse crime, to compute $u_{m}$ induced by targeted control parameters, the fluid-solid domain is discretized by using an element size of $0.1 \mathrm{~m}$, while an element size of $0.2 \mathrm{~m}$ is used for computing $u$ due to estimated control parameters. In the forward and inverse modeling, the time step is $0.001 \mathrm{~s}$, and the total observation duration $T$ is set as $1 \mathrm{~s}$.

In the following, we present three examples of numerical experiments. The first one tests the inversion performance of the three-level GA approach by using a dynamic force of a Ricker pulse signal with its central frequency of 5,10 , and $15 \mathrm{~Hz}$, respectively, in each level. In the second example, we examine the inversion performance by using a decreasing frequency-continuation counterpart of 15,10 , and $5 \mathrm{~Hz}$. The last example investigates the utilization of preliminary inversion using all-solid layers for detecting the presence of a fluidfilled cavity by identifying $E$ and $\rho$ of all the solid layers, among which one layer shows a very large value of $E$ (with a value being similar to that of the bulk modulus of water) and $\rho$ of the value of the mass density of water. Once the preliminary inversion is completed, our optimizer uses the presented three-level GA approach based on the fluid-solid wave model as a primary inversion.

To analyze the inversion results, the error between each target control parameter $\left(L_{s 1}, L_{f}, E_{s 1}\right.$, and $\left.E_{s 2}\right)$ and its estimated counterpart of the fittest individual at each generation is calculated as:

$$
\mathcal{E}=\frac{\mid \text { A targeted value }- \text { An estimated value } \mid}{\mid \text { A targeted value } \mid} \times 100[\%]
$$

We also calculate an averaged error of all the control parameters of the best-fit individual at each generation as:

$$
\overline{\mathcal{E}}=\frac{\sum_{k=1}^{4} \mathcal{E}_{k}}{4}[\%] .
$$

where $\mathcal{E}_{k}$ is the error (30) of the reconstruction of the $k$-th control parameter.

\subsection{Exemplary forward wave responses}

Prior to the study of the inversion performance, we show an exemplary forward wave response in the computational domain induced by a pulse loading at the top surface. We consider the fluid-solid model 3 with $L_{s 1}=50 \mathrm{~m}, L_{f}=80 \mathrm{~m}, L_{s 2}=120 \mathrm{~m}, E_{s 1}=2 \times 10^{8} \mathrm{~N} / \mathrm{m}^{2}$, and $E_{s 2}=5 \times 10^{8} \mathrm{~N} / \mathrm{m}^{2}$. The time-dependent value of the dynamic force applied at the top surface is a Ricker wavelet with its central frequency of $20 \mathrm{~Hz}$ and its peak amplitude of $1000 \mathrm{~N} / \mathrm{m}^{2}$.

The wave responses over space and time are shown in Fig. 3. In this seismogram, we show the stress field in the solid layers and the acoustic pressure in the fluid layer. The seismogram shows the following behaviors. (i) The elastic wave propagates throughout the first solid layer from the top surface and is transmitted via the first solid-fluid interface at $x=50 \mathrm{~m}$ to the fluid layer (please see the wave response around $x=50 \mathrm{~m}$ and $t=0.2 \mathrm{~s}$ ). While the wave enters into the fluid layer, it reflects off the interface back to the solid layer at the same time. ( $\mathrm{i}$ i) The acoustic pressure wave in the fluid layer is transmitted into the second solid layer through the second solid-fluid interface at $x=80 \mathrm{~m}$ and reflects off the interface back to the fluid layer (please see the wave response around $x=80 \mathrm{~m}$ and $t=0.2 \mathrm{~s}$ ). (iii) The stress wave in the second solid layer is transmitted through the absorbing boundary at $x=120 \mathrm{~m}$ without any reflection (please see the wave response around $x=120 \mathrm{~m}$ and $t=0.3 \mathrm{~s}$ ). The system repeats $(i)$ to $(i i i)$ until the amplitude of the wave fades away.

In the presented inverse modeling, the sensor on the top surface records the response signal in the time domain. The amplitudes and timings of the recorded signal may provide the inversion solver with information about the material properties of solid layers and the locations of fluid-solid interfaces. In other words, the timings of particular parts of the signal could indicate, primarily, the locations $\left(L_{s 1}\right.$ and $\left.L_{f}\right)$ of the fluid-solid interfaces, while the amplitudes of particular parts of the signal could indicate, primarily, the material properties $\left(E_{s 1}\right.$ and $\left.E_{s 2}\right)$ in the solid layers. 


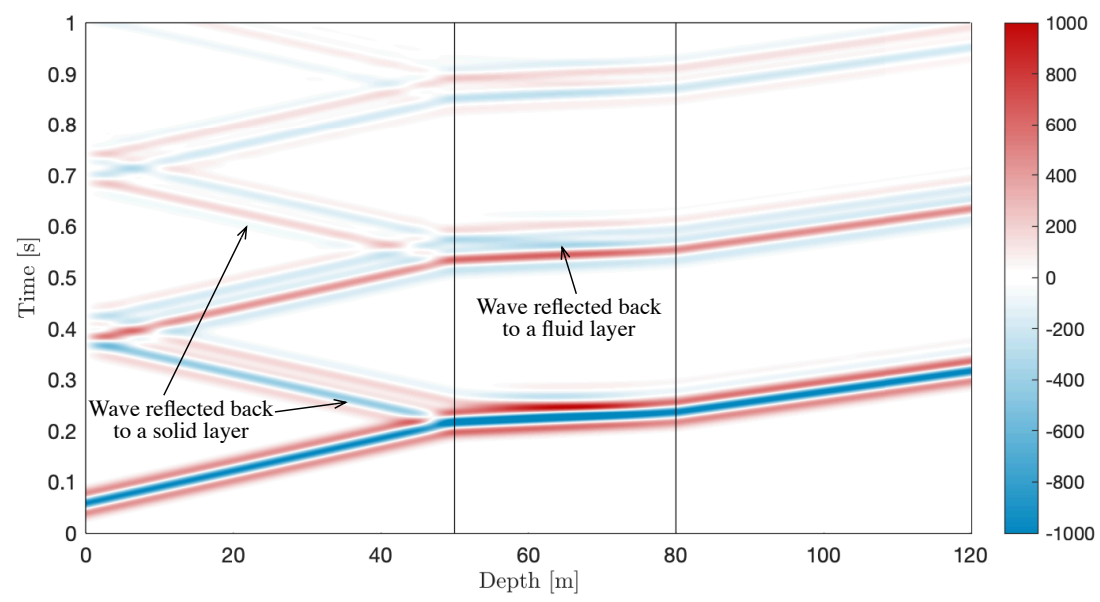

Figure 3: The seismogram of the stress field $\left[\mathrm{N} / \mathrm{m}^{2}\right]$ in the solid layers $(0 \leq x \leq 50$ and $80 \leq x)$ and the acoustic pressure $\left[\mathrm{N} / \mathrm{m}^{2}\right]$ in the fluid layer $(50<x<80)$ induced by a dynamic loading at the top surface $(x=0)$. The solid lines at $x=50$ and $80 \mathrm{~m}$ indicate the fluid-solid interfaces. The arrows indicate the waves that are reflected from the interfaces and propagating backward. The acoustic waves that are reflected from the second interface are more visible in the time of $0.55 \mathrm{~s}$ than in $0.25 \mathrm{~s}$ in this plot.

\subsection{Example 1 - Investigating the inversion performance by increasing the dominant frequency of excitation for each GA level}

In this example, we study the performance of the presented multi-level GA for identifying targeted control parameters by increasing the frequency of an excitational Ricker signal for each GA level. That is, a dynamic pulse of its dominant frequency $5 \mathrm{~Hz}$ is used in the first-level GA, and, then, 10 and $20 \mathrm{~Hz}$ are used in the next levels. The control parameters to be identified are $L_{s 1}, L_{f}, E_{s 1}$, and $E_{s 2}$. We considered Cases 1-3, which use the fluid-solid models 1,2 , and 3 , respectively.

- In Case 1, the targeted values of $L_{s 1}$ and $L_{f}$ are 20 and $25 \mathrm{~m}$, respectively, while the values of their estimated counterparts, during the first-level GA level, are bounded as $10 \leq L_{s 1} \leq 22 \mathrm{~m}$ and 23 $\leq L_{f} \leq 35 \mathrm{~m}$. Please note that their upper and lower limits are changed for the second and third GA levels as previously discussed.

- In Case 2, the targeted values of $L_{s 1}$ and $L_{f}$ are set to be 40 and $45 \mathrm{~m}$, respectively, while the values of their estimated counterparts, during the first GA level, are bounded as $30 \leq L_{s 1} \leq 42 \mathrm{~m}$ and 43 $\leq L_{f} \leq 55 \mathrm{~m}$.

- In Case 3, the estimated values of $L_{s 1}$ and $L_{f}$ bounded as $40 \leq L_{s 1} \leq 65 \mathrm{~m}$ and $66 \leq L_{f} \leq 90 \mathrm{~m}$ in the first-level GA, and their targeted counterparts are 50 and $80 \mathrm{~m}$, respectively.

For all cases, the targeted values of Young's moduli are set to be $E_{s 1}=2 \times 10^{8} \mathrm{~Pa}$ and $E_{s 2}=5 \times 10^{8} \mathrm{~Pa}$, while their estimated values for both moduli during the first GA level are bounded as $1 \times 10^{8}$ Pa $\leq E \leq$ $7.5 \times 10^{8} \mathrm{~Pa}$. Their upper and lower limits are changed for the second and third GA levels as previously discussed.

Table 1 presents the reconstructed control parameters of the fittest individual that is obtained at the last generation of each GA level per each case. In the table, we also present the average error $\overline{\mathcal{E}}$, (31), of all the parameters reconstructed at the end of each GA level and the error $\mathcal{E}$, (30), of each control parameter of the fittest individual at the end of the last-level GA. In all Cases 1 to 3 , the value of $\overline{\mathcal{E}}$ at the end of the last-level GA is smaller than that at the end of the first-level GA, and the final values of $\overline{\mathcal{E}}$ in all the cases 1 to 3 are smaller than $1 \%$.

Fig. 4 shows that the average error for the best-fit individual at each generation tends to be decreased during the presented inversion process. We note that, after about 20 GA iterations in the first-level GA, the value of $\overline{\mathcal{E}}$ is converged but still shows room for improvement (i.e., the optimizer finds an inversion solution around a local minimum). To address such solution multiplicity in the first-level GA, we use a misfit function for $u_{m}$ induced by a pulse signal of a new central frequency in the next-level GA. By doing so, the optimizer in the second-level GA is able to escape the local minimum of the first-level GA. By applying the same technique in the third-level GA, we can resolve the solution multiplicity of the second-level GA. Therefore, 
Table 1: Example 1: Reconstructed values of the control parameters for each GA level obtained by increasing the frequency of the dynamic force for each GA level. The last row of each case represents the error at the third-level GA.

\begin{tabular}{lcccccc}
\hline Cases & GA & $L_{s 1}$ & $\begin{array}{c}L_{f} \\
(\mathrm{~m})\end{array}$ & $\begin{array}{c}E_{s 1} \\
(\mathrm{~Pa})\end{array}$ & $\begin{array}{c}E_{s 2} \\
(\mathrm{~Pa})\end{array}$ & $\begin{array}{c}\text { Average Error } \\
\overline{\mathcal{E}}\end{array}$ \\
\hline Case 1 & Target & 20 & 25 & $2 \times 10^{8}$ & $5 \times 10^{8}$ & \\
& 1st & 20.4 & 24.9 & $2.13 \times 10^{8}$ & $5.66 \times 10^{8}$ & $5.53 \%$ \\
& 2nd & 19.8 & 24.3 & $1.97 \times 10^{8}$ & $4.94 \times 10^{8}$ & $1.63 \%$ \\
& 3rd & 20.0 & 24.8 & $2.00 \times 10^{8}$ & $5.08 \times 10^{8}$ & $0.60 \%$ \\
& Individual error & $0.00 \%$ & $0.80 \%$ & $0.00 \%$ & $1.60 \%$ & \\
\hline Case 2 & Target & 40 & 45 & $2 \times 10^{8}$ & $5 \times 10^{8}$ & \\
& 1st & 41.7 & 46.2 & $2.20 \times 10^{8}$ & $5.86 \times 10^{8}$ & $8.53 \%$ \\
& 2nd & 40.6 & 46.0 & $2.06 \times 10^{8}$ & $5.16 \times 10^{8}$ & $2.48 \%$ \\
& 3rd & 39.9 & 44.7 & $1.98 \times 10^{8}$ & $4.97 \times 10^{8}$ & $0.63 \%$ \\
& Individual error & $0.25 \%$ & $0.67 \%$ & $1.00 \%$ & $0.60 \%$ & \\
\hline Case 3 & Target & 50 & 80 & $2 \times 10^{8}$ & $5 \times 10^{8}$ & \\
& 1st & 53.1 & 90.0 & $2.28 \times 10^{8}$ & $5.49 \times 10^{8}$ & $10.63 \%$ \\
& 2nd & 48.9 & 79.2 & $1.91 \times 10^{8}$ & $5.33 \times 10^{8}$ & $3.58 \%$ \\
& 3rd & 49.9 & 79.9 & $1.98 \times 10^{8}$ & $5.06 \times 10^{8}$ & $0.63 \%$ \\
& Individual error & $0.20 \%$ & $0.12 \%$ & $1.00 \%$ & $1.20 \%$ & \\
\hline
\end{tabular}

we suggest that it is feasible to reconstruct control parameters effectively by using a multi-level GA process combined with a frequency-continuation scheme, by which the frequency is progressively increased for each GA level.

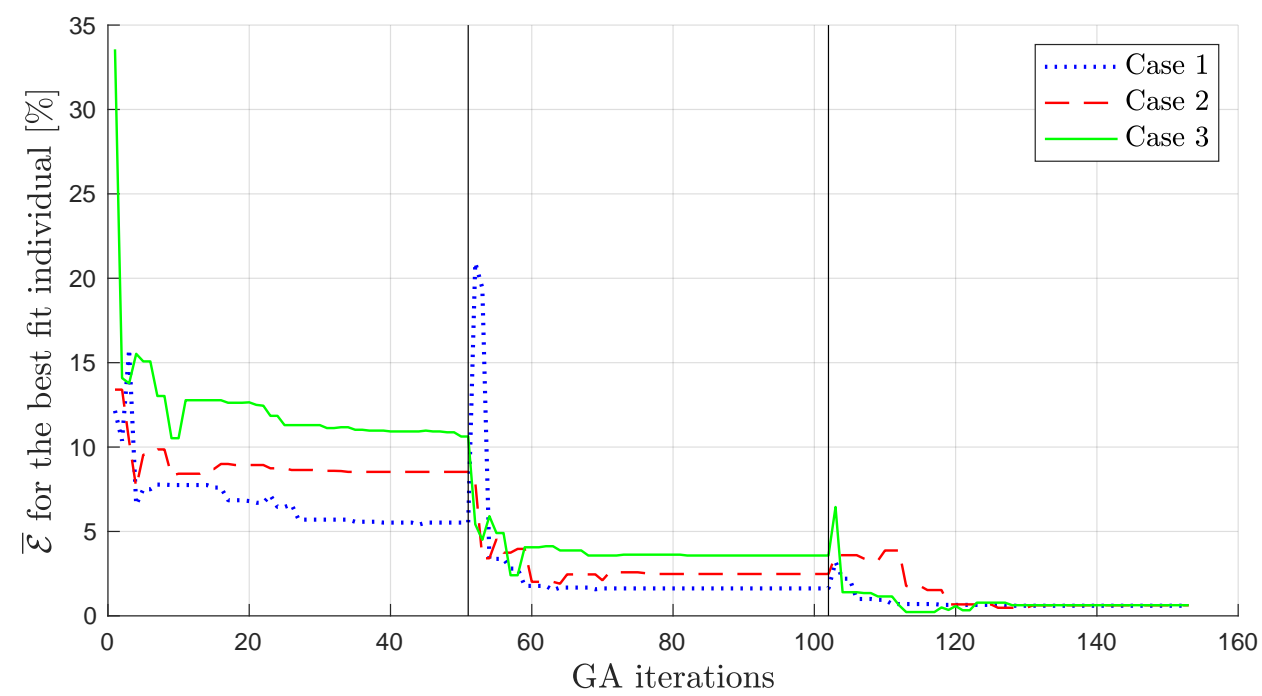

Figure 4: $\overline{\mathcal{E}}$ for the best-fit individual versus the GA iteration in Example 1. The vertical solid lines at the 51st and 102nd GA iteration indicate the starting of a new GA level and the change in the frequency of the excitational Ricker signal.

Fig. 5 presents the detail of the inversion performance of Case 3. Namely, Fig. 5 shows the histograms of the estimated control parameters of the entire population of the individuals during all the GA levels in Case 3. During the initial few generations of each GA level, the inversion solver explores a broader range of estimated values of control parameters. After these initial generations, the values of estimated parameters of individuals are converged.

Fig. 6 also shows that $u_{m}$ induced by the target parameters matches $u$ due to the finally-reconstructed control parameters, obtained at the end of each GA set, at the sensor on the top surface when a Ricker signal with its central frequency of $5 \mathrm{~Hz}, 10 \mathrm{~Hz}$, or $20 \mathrm{~Hz}$ is employed in Case 3. 

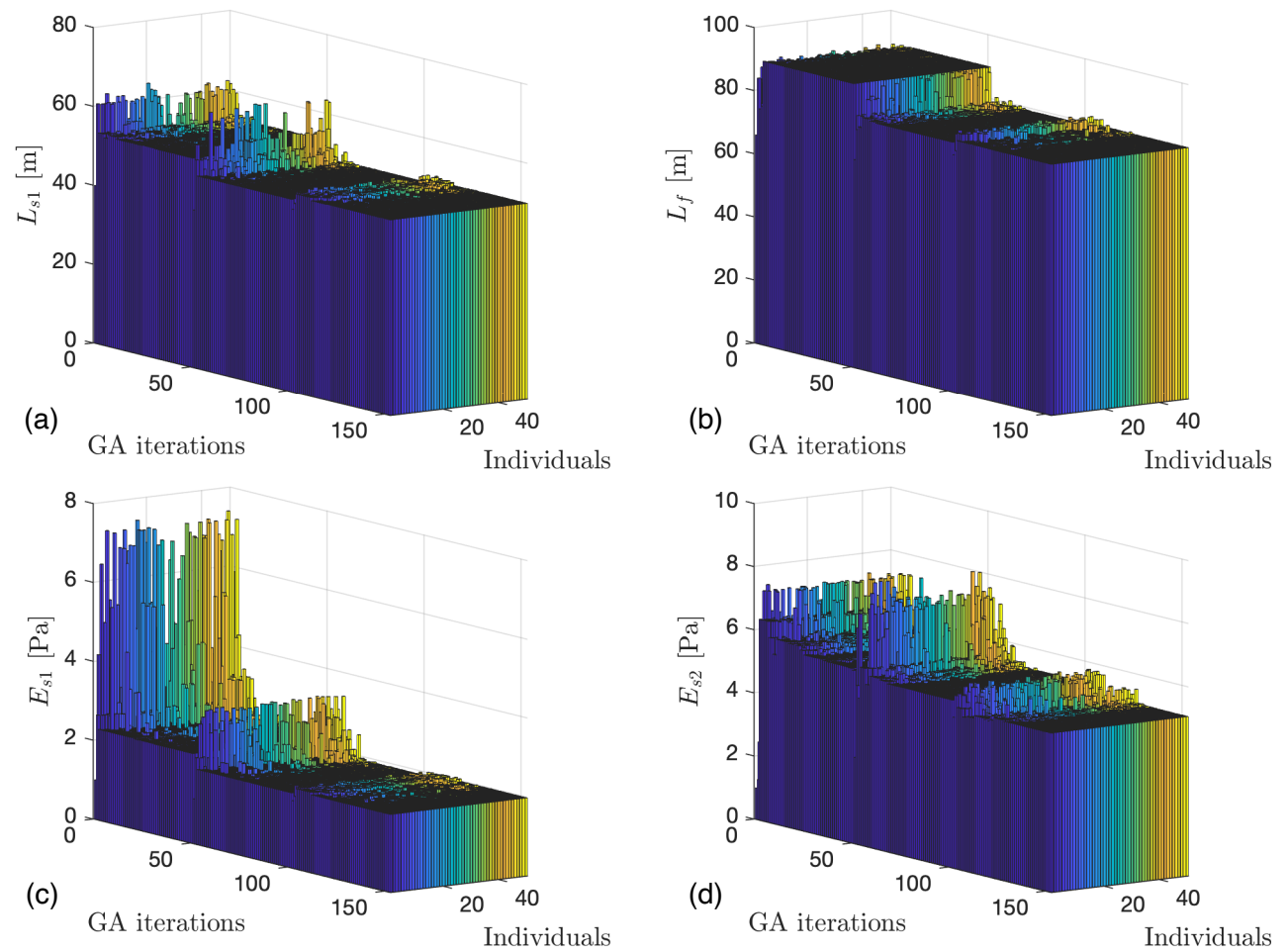

Figure 5: The histograms of (a) $L_{s 1}$, (b) $L_{f}$, (c) $E_{s 1}$, and (d) $E_{s 2}$ of the entire population of the individuals at all the generations in Case 3.
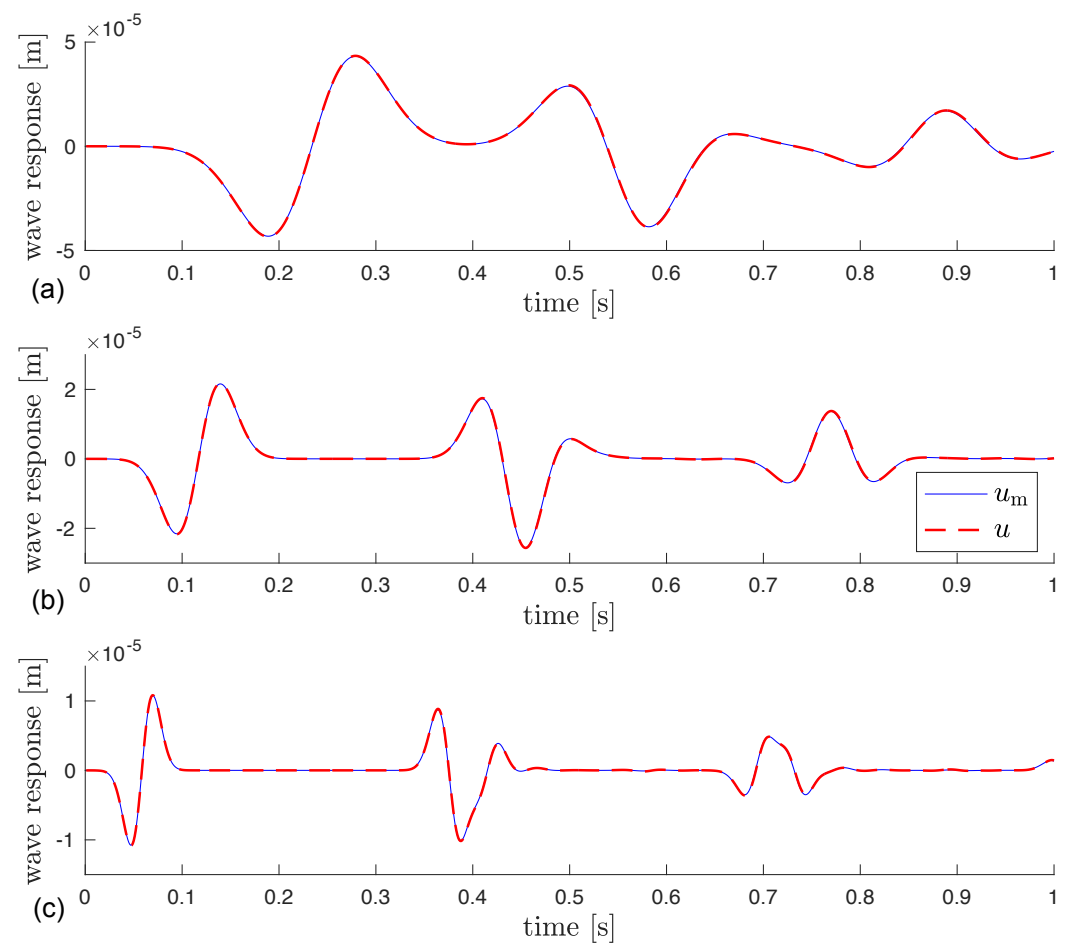

Figure 6: Wave responses, $u_{m}$ and $u$, at the sensor on the top surface induced by a pulse signal of (a) $5 \mathrm{~Hz}$, (b) $10 \mathrm{~Hz}$, and (c) $20 \mathrm{~Hz}$ in Case 3. 


\subsection{Example 2 - Investigating the inversion performance by decreasing the dominant frequency of excitation for each GA level}

This example studies the performance of reconstructing the control parameters by using the presented multi-level GA combined with a frequency-continuation scheme that decreases the dominant frequency of the dynamic pulse over the multiple GA levels. The first, second, and third-level GA use input force signals of central frequencies of $20 \mathrm{~Hz}, 10 \mathrm{~Hz}$, and $5 \mathrm{~Hz}$, respectively. We examined Cases 4-6, which use the models 1, 2, and 3, respectively. The upper and lower limits of estimated control parameters for each GA level are set the same as those in Example 1. Table 2 shows the values of finally-reconstructed control parameters, and Fig. 7 shows that the average errors for the best-fit individual of all the Cases 4-6 become smaller over the generations and the GA levels. The final values of $\overline{\mathcal{E}}$ in all the Cases 4 to 6 are smaller than $2 \%$ in Example 2. Thus, we suggest that the presented multi-level GA-based optimizer combined with a frequency-continuation scheme, which decreases the frequency for each GA level, is able to identify the values of the targeted control parameters as effectively as the increasing-frequency counterpart. However, this would be the case only in the presented example because the presented example contains a small number of control parameters. In a more complex case (e.g., 2D or 3D settings), where there are a large number of control parameters, the decreasing frequency-continuation scheme may not be as effective as its counterpart of increasing frequency.

Table 2: Example 2: Reconstructed values of the control parameters for each GA level obtained by decreasing the frequency of the dynamic force for each GA level.

\begin{tabular}{lcccccc}
\hline Cases & GA & $L_{s 1}$ & $\begin{array}{c}L_{f} \\
(\mathrm{~m})\end{array}$ & $\begin{array}{c}E_{s 1} \\
(\mathrm{~Pa})\end{array}$ & $\begin{array}{c}E_{s 2} \\
(\mathrm{~Pa})\end{array}$ & $\begin{array}{c}\text { Average Error } \\
\overline{(\mathrm{m})}\end{array}$ \\
\hline Case 4 & Target & 20 & 25 & $2 \times 10^{8}$ & $5 \times 10^{8}$ & \\
& 1st & 21.4 & 27.3 & $2.29 \times 10^{8}$ & $5.55 \times 10^{8}$ & $10.43 \%$ \\
& 2nd & 20.1 & 24.9 & $2.04 \times 10^{8}$ & $5.18 \times 10^{8}$ & $1.63 \%$ \\
& 3rd & 20.2 & 25.7 & $2.02 \times 10^{8}$ & $5.04 \times 10^{8}$ & $1.40 \%$ \\
& Individual error & $1.00 \%$ & $2.80 \%$ & $1.00 \%$ & $0.80 \%$ & \\
\hline Case 5 & Target & 40 & 45 & $2 \times 10^{8}$ & $5 \times 10^{8}$ & \\
& 1st & 41.0 & 45.7 & $2.11 \times 10^{8}$ & $6.01 \times 10^{8}$ & $7.44 \%$ \\
& 2nd & 40.6 & 46.3 & $2.05 \times 10^{8}$ & $5.09 \times 10^{8}$ & $2.17 \%$ \\
& 3rd & 40.0 & 44.7 & $2.00 \times 10^{8}$ & $5.01 \times 10^{8}$ & $0.22 \%$ \\
& Individual error & $0.00 \%$ & $0.67 \%$ & $0.00 \%$ & $0.20 \%$ & \\
\hline Case 6 & Target & 50 & 80 & $2 \times 10^{8}$ & $5 \times 10^{8}$ & \\
& 1st & 57.5 & 82.3 & $2.63 \times 10^{8}$ & $4.51 \times 10^{8}$ & $14.79 \%$ \\
& 2nd & 49.7 & 77.9 & $1.98 \times 10^{8}$ & $4.88 \times 10^{8}$ & $1.66 \%$ \\
& 3rd & 49.7 & 79.9 & $1.99 \times 10^{8}$ & $5.18 \times 10^{8}$ & $1.21 \%$ \\
& Individual error & $0.60 \%$ & $0.12 \%$ & $0.50 \%$ & $3.60 \%$ & \\
\hline
\end{tabular}

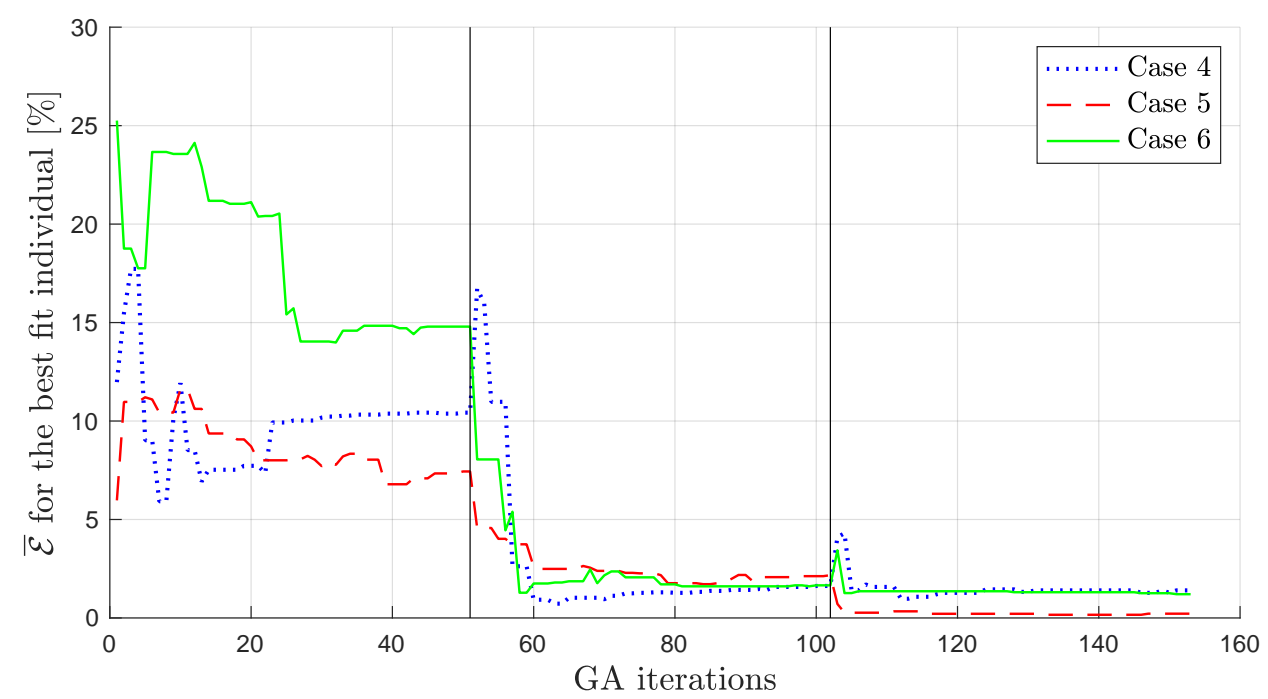

Figure 7: $\overline{\mathcal{E}}$ for the best-fit individual versus the GA iteration in Example 2. The solid lines at the 51st and 102nd GA iteration indicate the starting of a new GA level and the change in frequency. 


\subsection{Example 3 - Utilization of a preliminary inversion using only solid layers for detect- ing the presence of a fluid layer, followed by a primary inversion using fluid-solid layers}

In this example, we present a preliminary inversion using only solid layers for identifying if a target model contains a fluid-filled cavity. Here, three target models are used: one, which does not contain a fluid layer, and the other two, in which a fluid-filled cavity is included. For all models, our preliminary GA parameterestimation method assumes that the target contains only solid layers. Once the preliminary inversion is completed, we continue a primary inversion using a fluid-solid model in a manner that is similar to what is described in Example 1. Such a two-step (preliminary to primary inversion) approach is applied to the following Cases 7-10.

- Cases 7 and 8 consider a target Model 4, which does not include a fluid layer, and contains only three solid layers of $5 \mathrm{~m}$ length each. The targeted Young's modulus of each layer is: $E_{s 1}=2 \times 10^{8} \mathrm{~Pa}, E_{s 2}=$ $3 \times 10^{8} \mathrm{~Pa}$, and $E_{s 3}=5 \times 10^{8} \mathrm{~Pa}$, respectively. For Case 7, the targeted mass densities of all the layers are all same: $\rho_{s 1}=\rho_{s 2}=\rho_{s 3}=2000 \mathrm{~kg} / \mathrm{m}^{3}$, whereas, for Case 8 , they are all different from each other: $\rho_{s 1}=1500 \mathrm{~kg} / \mathrm{m}^{3}, \rho_{s 2}=2000 \mathrm{~kg} / \mathrm{m}^{3}$, and $\rho_{s 3}=2200 \mathrm{~kg} / \mathrm{m}^{3}$.

- Case 9 considers Model 5, which consists of three layers, i.e., two solids and one fluid. Model 5 has a total length $L_{s 2}$ of $15 \mathrm{~m}$, and its fluid is located between $x$ of $L_{s 1}=5 \mathrm{~m}$ and $x$ of $L_{f}=10 \mathrm{~m}$. The Young's moduli of the solid layers are $E_{s 1}=2 \times 10^{8} \mathrm{~Pa}$ and $E_{s 2}=5 \times 10^{8}$, and their mass densities are $\rho_{s 1}=\rho_{s 2}=2000 \mathrm{~kg} / \mathrm{m}^{3}$, respectively. The bulk modulus $\left(k_{f}\right)$ and mass density $\left(\rho_{f}\right)$ of the fluid layer are $2.34 \times 10^{9} \mathrm{~N} / \mathrm{m}^{2}$ and $1021 \mathrm{~kg} / \mathrm{m}^{3}$, respectively.

- Case 10 considers Model 6, which consists of a fluid layer surrounded by solid layers. The fluid layer is located between $x$ of $L_{s 1}=4 \mathrm{~m}$ and $x$ of $L_{f}=9.3 \mathrm{~m}$, and its $L_{s 2}$ is $15 \mathrm{~m}$. The properties of the fluid layer $\left(k_{f}\right.$ and $\left.\rho_{f}\right)$ and the Young's moduli of the solid layers $\left(E_{s 1}\right.$ and $\left.E_{s 2}\right)$ are the same as those in Case 9 except that the mass densities of solid layers are $\rho_{s 1}=1900 \mathrm{~kg} / \mathrm{m}^{3}$ and $\rho_{s 2}=2100 \mathrm{~kg} / \mathrm{m}^{3}$.

In Case 7 and 8, we study the capability of the preliminary inversion to detect the absence of a fluid layer by estimating $E$ and $\rho$ of all solid layers. In Case 9 and 10, by using only solid layers during the preliminary inversion, we investigate what values of final-converged $E$ and $\rho$ from the preliminary inversion indicate the presence of a fluid-filled cavity in the model.

\subsubsection{Preliminary inversion using all solid-layer model}

For all Cases 7-10, the preliminary inversion method utilizes only one frequency level of GA, where a Ricker pulse signal with a central frequency of $50 \mathrm{~Hz}$ is used as the dynamic force, and the number of generations and population size are 50 and 100, respectively. Moreover, the estimated values for Young's moduli in all solid layers are bounded as $1 \times 10^{8} \leq E \leq 50 \times 10^{8} \mathrm{~Pa}$, and the estimated values of their $\rho$ bounded as $1000 \leq \rho \leq 3000 \mathrm{~kg} / \mathrm{m}^{3}$. Furthermore, the preliminary inversion assumes that the target contains three solid layers of $5 \mathrm{~m}$ length each.

Fig. 8 shows the reduction of average errors for the best-fit individual for Cases 7 to 10 as the number of GA generations increases in the preliminary inversion. Table 3 shows the targeted and reconstructed control parameters from the preliminary inversion for Cases 7 to 10. For Cases 7 and 8, the results show that the preliminary GA-based inversion algorithm can determine that all the layers in the target model are solid layers because the order of magnitude of estimated Young's moduli are all 2 to $5 \times 10^{8} \mathrm{~Pa}$ in all the solid layers, and the order of magnitude of their estimated mass densities are all 1500 to $2200 \mathrm{~kg} / \mathrm{m}^{3}$. The average error in Cases 7 and 8 is 3.82\% and 4.82\%, respectively. On the contrary, in Cases 9 and 10, the preliminary inversion led to the estimated values of $E_{s 2}$ and $\rho_{s 2}$ of the second solid layer such that it can be interpreted as a fluid layer. Namely, the estimated values of $E_{s 2}$ as $22.95 \times 10^{8} \mathrm{~Pa}$ (Case 9) and $24.32 \times 10^{8}$ $\mathrm{Pa}$ (Case 10) are similar to the bulk modulus $23.4 \times 10^{8} \mathrm{~Pa}$ of water, and the estimated values of $\rho_{s 2}$ as 1022 $\mathrm{kg} / \mathrm{m}^{3}$ (Case 9) and $1070 \mathrm{~kg} / \mathrm{m}^{3}$ (Case 10) are similar to the mass density $1021 \mathrm{~kg} / \mathrm{m}^{3}$ of water. The average errors in Cases 9 and 10 are $2.16 \%$ and 17.13\%, respectively. Thus, after detecting the fluid layers in Case 9 and 10, we continue the primary inversion in Cases 9 and 10 as follows. 
Table 3: Example 3: Reconstructed values of the control parameters obtained by an preliminary inversion. In Cases 9 and 10 , the targeted $E_{s 2}{ }^{*}\left(23.4 \times 10^{8} \mathrm{~Pa}\right)$ adopts the bulk modulus of the fluid layer.

\begin{tabular}{|c|c|c|c|c|c|c|c|c|}
\hline Cases & & $\begin{array}{l}E_{s 1} \\
(\mathrm{~Pa})\end{array}$ & $\begin{array}{l}E_{s 2} \\
(\mathrm{~Pa})\end{array}$ & $\begin{array}{l}E_{s 3} \\
(\mathrm{~Pa})\end{array}$ & $\begin{array}{c}\rho_{s 1} \\
\left(\mathrm{~kg} / \mathrm{m}^{3}\right)\end{array}$ & $\begin{array}{c}\rho_{s 2} \\
\left(\mathrm{~kg} / \mathrm{m}^{3}\right)\end{array}$ & $\begin{array}{c}\rho_{s 3} \\
\left(\mathrm{~kg} / \mathrm{m}^{3}\right)\end{array}$ & $\begin{array}{l}\text { Average } \\
\text { Error } \overline{\mathcal{E}}\end{array}$ \\
\hline \multirow{3}{*}{$\begin{array}{l}\text { Case } \\
7\end{array}$} & Target & $2 \times 10^{8}$ & $3 \times 10^{8}$ & $5 \times 10^{8}$ & 2000 & 2000 & 2000 & \\
\hline & Preliminarv values & $201 \times 10^{8}$ & $288 \times 10^{8}$ & $526 \times 10^{8}$ & 1943 & 2017 & 1810 & $302 \%$ \\
\hline & Individual error & $0.50 \%$ & $4.00 \%$ & $5.20 \%$ & $2.85 \%$ & $0.85 \%$ & $9.50 \%$ & \\
\hline \multirow{4}{*}{$\begin{array}{l}\text { Case } \\
8\end{array}$} & Target & $2 \times 10^{8}$ & $3 \times 10^{8}$ & $5 \times 10^{8}$ & 1500 & 2000 & 2200 & \\
\hline & & & & & & & & \\
\hline & Preliminary values & $2.10 \times 10^{8}$ & $3.08 \times 10^{8}$ & $4.93 \times 10^{8}$ & 1560 & 2092 & 2448 & $4.82 \%$ \\
\hline & Individual error & $5.00 \%$ & $2.67 \%$ & $1.40 \%$ & $4.00 \%$ & $4.60 \%$ & $11.27 \%$ & \\
\hline \multirow{4}{*}{$\begin{array}{l}\text { Case } \\
9\end{array}$} & Target & $2 \times 10^{8}$ & $23.40 \times 10^{8 *}$ & $5 \times 10^{8}$ & 2000 & 1021 & 2000 & \\
\hline & & & & & & & & \\
\hline & Preliminary values & $2.01 \times 10^{8}$ & $22.95 \times 10^{8}$ & $5.07 \times 10^{8}$ & 2024 & 1022 & 1843 & $2.16 \%$ \\
\hline & Individual error & $0.50 \%$ & $1.92 \%$ & $1.40 \%$ & $1.20 \%$ & $0.10 \%$ & $7.85 \%$ & \\
\hline \multirow{3}{*}{$\begin{array}{l}\text { Case } \\
10\end{array}$} & Target & $2 \times 10^{8}$ & $23.40 \times 10^{8 *}$ & $5 \times 10^{8}$ & 1900 & 1021 & 2100 & \\
\hline & Preliminary values & $2.52 \times 10^{8}$ & $24.32 \times 10^{8}$ & $6.53 \times 10^{8}$ & 1549 & 1070 & 1701 & $17.13 \%$ \\
\hline & Individual error & $26.00 \%$ & $3.93 \%$ & $30.60 \%$ & $18.47 \%$ & $4.80 \%$ & $19.00 \%$ & \\
\hline
\end{tabular}

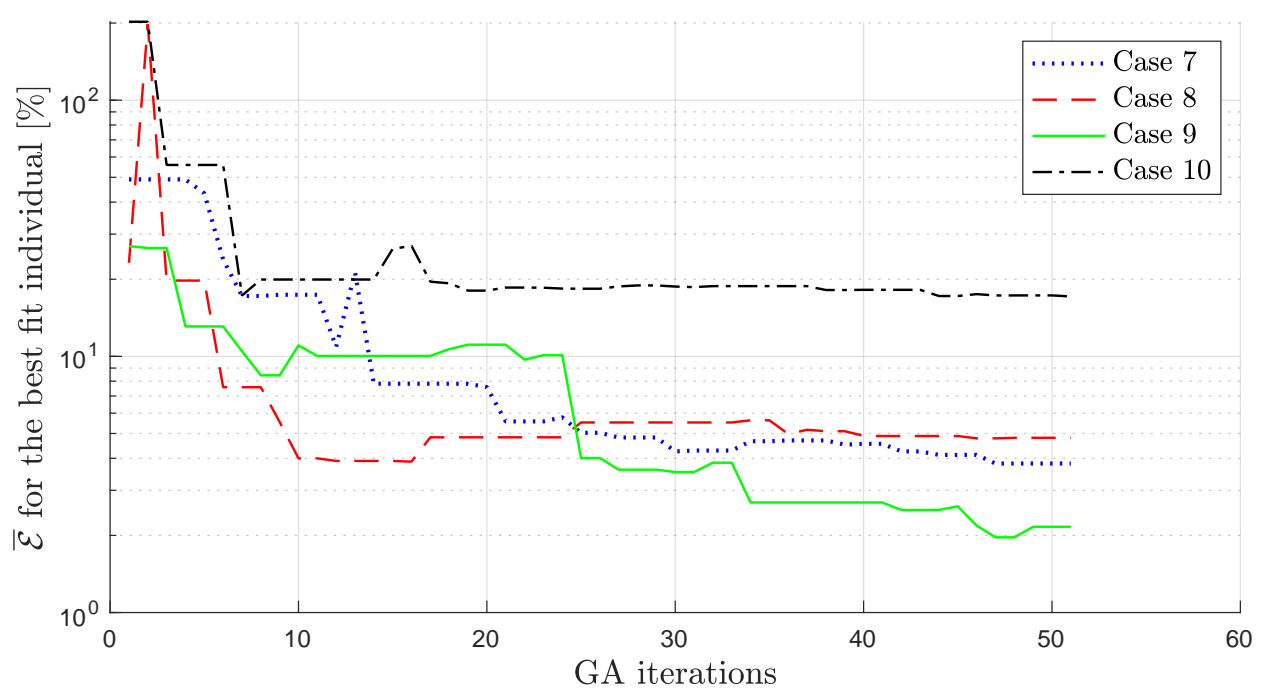

Figure 8: $\overline{\mathcal{E}}$ for the best-fit individual versus the GA iteration in Example 3 for the preliminary inversion.

\subsubsection{Primary inversion using a fluid-solid model}

After the preliminary inversion using the solid-only wave solver, the three frequency-level GA-based optimizer that uses a fluid-solid wave model estimates the fluid layer's depth information and the material properties $(E$ and $\rho$ ) of the soil layers. Namely, the preliminary inversion results from Cases 9 and 10 are used for determining the bounds of guessed parameters in the first-frequency set of the primary GA inversion using the fluid-solid model. The increasing-frequency continuation approach is utilized with a dynamic pulse of its dominant frequency $5 \mathrm{~Hz}$ in the first frequency-level GA and, then, 10 and $20 \mathrm{~Hz}$ in the next frequency levels while the values of the estimated parameters are bounded in each frequency set in the following manner.

- During the first frequency-level GA using the fluid-solid wave solver, the estimated $L_{s 1}$ and $L_{f}$ values are bounded as $2 \leq L_{s 1} \leq 7 \mathrm{~m}$ and $8 \leq L_{f} \leq 13 \mathrm{~m}$, the estimated values of $E_{s 1}$ and $E_{s 2}$ are bounded as $1 \times 10^{8} \leq E \leq 7.5 \times 10^{8} \mathrm{~Pa}$, and the estimated values of $\rho_{s 1}$ and $\rho_{s 2}$ (i.e., the mass densities of surrounding soil layers) are set as $1500 \leq \rho \leq 2500 \mathrm{~kg} / \mathrm{m}^{3}$.

- In the second frequency-level GA, the values of estimated $L_{s 1}$ and $L_{f}$ are bounded using the same 
upper and lower limits as the first frequency-level GA. The values of estimated $E_{s 1}$ and $E_{s 2}$ are bounded using $\pm 50 \%$ derivations of their final-reconstructed values obtained during the first frequencylevel GA, while the values of estimated $\rho_{s 1}$ and $\rho_{s 2}$ are bounded using $\pm 20 \%$ derivations.

- Finally, in the last frequency-level GA, the estimated $L_{s 1}$ and $L_{f}$ values are bounded by using \pm $5 \%$ derivations of their final-reconstructed values obtained during the second frequency-level GA, while $\pm 10 \%$ derivations of the values of estimated $E_{s 1}$ and $E_{s 2}$ are employed to bound the limits of Young's moduli, and $\pm 10 \%$ derivations of the final-reconstructed values of $\rho_{s 1}$ and $\rho_{s 2}$ are used as theirs bounds in the last frequency-level GA.

Table 4 shows the values of finally-reconstructed control parameters in Cases 9 and 10. For Case 9, the value of $\overline{\mathcal{E}}$ at the end of the last-level GA, $1.74 \%$, is smaller than the error obtained in the preliminary result, $2.16 \%$, shown in Table 3. The same can be noted in Case 10, where $\overline{\mathcal{E}}$ at the end of the last-level GA, $2.79 \%$, is much smaller than $\overline{\mathcal{E}}$ from the preliminary inversion, $17.13 \%$. Fig. 9 shows the average error for the best-fit individual over GA iterations of the three-level primary GA inversion using fluid-solid models for both Cases 9 and 10. During the initial several iterations of each frequency level, the algorithm focuses on exploring widely-varying values of the estimated control parameters within the given limits, and this behavior increases error. Later, the error of the algorithm is converged by finding better-fit individuals. Fig. 10 shows the histograms of the estimated control parameters of the entire population of the individuals during all the GA levels in Case 10 during the primary inversion. It demonstrates that, at the beginning of each frequency level, GA explores wide ranges of control parameters and eventually converges to the targeted parameters at the end of the last frequency set.

Table 4: Example 3 - the results of the primary inversion in Cases 9 and 10: Estimated values of the control parameters in each GA level obtained by increasing the frequency of the dynamic force for each GA level.

\begin{tabular}{lcccccccc}
\hline Cases & $\begin{array}{c}\text { GA } \\
\text { level }\end{array}$ & $\begin{array}{c}L_{s 1} \\
(\mathrm{~m})\end{array}$ & $\begin{array}{c}L_{f} \\
(\mathrm{~m})\end{array}$ & $\begin{array}{c}E_{s 1} \\
(\mathrm{~Pa})\end{array}$ & $\begin{array}{c}E_{s 2} \\
(\mathrm{~Pa})\end{array}$ & $\begin{array}{c}\rho_{s 1} \\
\left(\mathrm{~kg} / \mathrm{m}^{3}\right)\end{array}$ & $\begin{array}{c}\rho_{s_{2}} \\
\left(\mathrm{~kg} / \mathrm{m}^{3}\right)\end{array}$ & $\begin{array}{c}\text { Average Error } \\
\overline{\mathcal{E}}\end{array}$ \\
\hline Case & Target & 5 & 10 & $2 \times 10^{8}$ & $5 \times 10^{8}$ & 2000 & 2000 & \\
9 & & & & & & & & \\
& 1st & 5.1 & 10.0 & $2.01 \times 10^{8}$ & $5.41 \times 10^{8}$ & 1965 & 1843 & $3.38 \%$ \\
& 2nd & 5.1 & 9.8 & $2.01 \times 10^{8}$ & $5.45 \times 10^{8}$ & 1969 & 1843 & $3.82 \%$ \\
& 3rd & 5.1 & 10.1 & $1.99 \times 10^{8}$ & $5.12 \times 10^{8}$ & 2011 & 1920 & $1.74 \%$ \\
& Individual error & $2.00 \%$ & $1.00 \%$ & $0.50 \%$ & $2.40 \%$ & $0.55 \%$ & $4.00 \%$ & \\
\hline Case & Target & 4 & 9.3 & $2 \times 10^{8}$ & $5 \times 10^{8}$ & 1900 & 2100 & \\
& & & & & & & & \\
& 1st & 3.1 & 8.4 .2 & $1.59 \times 10^{8}$ & $4.68 \times 10^{8}$ & 2403 & 2239 & $15.36 \%$ \\
& 2nd & 4.1 & 9.3 & $2.02 \times 10^{8}$ & $5.42 \times 10^{8}$ & 1922 & 1981 & $3.12 \%$ \\
& 3rd & 4.0 & 9.3 & $2.01 \times 10^{8}$ & $5.37 \times 10^{8}$ & 1922 & 1939 & $2.79 \%$ \\
& Individual error & $0.00 \%$ & $0.00 \%$ & $0.50 \%$ & $7.40 \%$ & $1.16 \%$ & $7.67 \%$ & \\
\hline
\end{tabular}




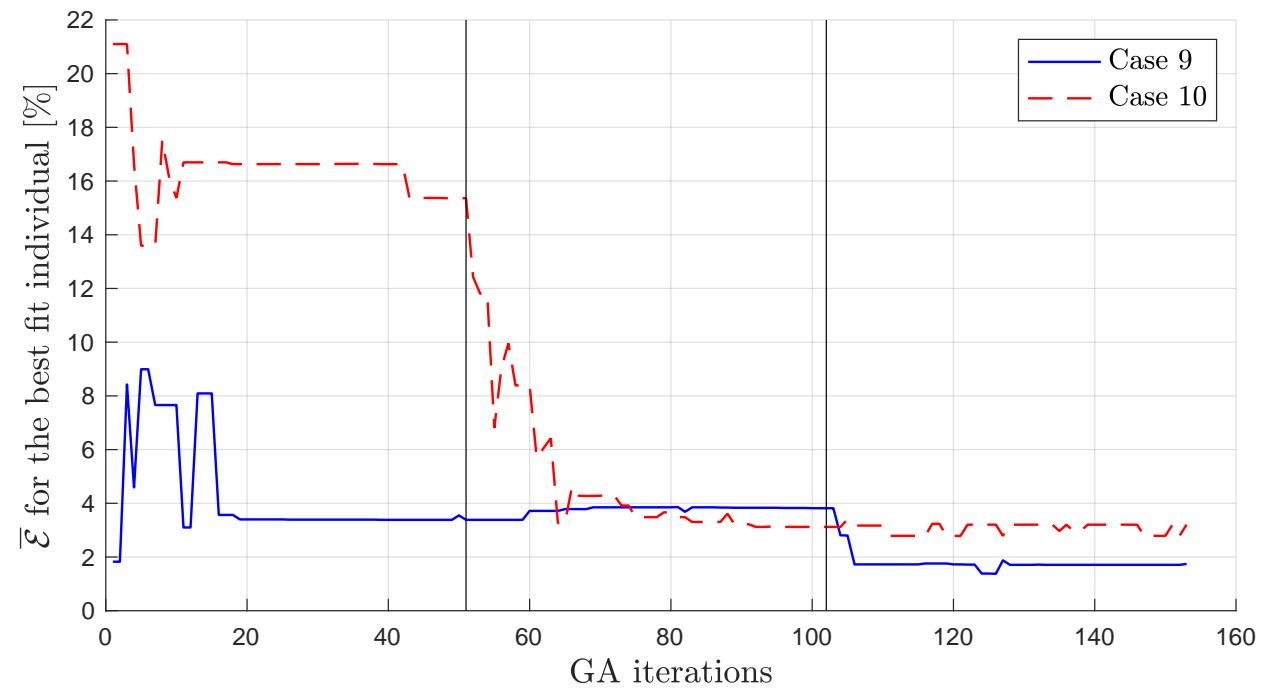

Figure 9: $\overline{\mathcal{E}}$ for the best-fit individual versus the GA iteration in Cases 9 and 10 during the primary inversion. The vertical solid lines at the 51st and 102nd GA iterations indicate each onset of a new frequency-level GA and the change in the frequency of the excitational Ricker signal.

(a)

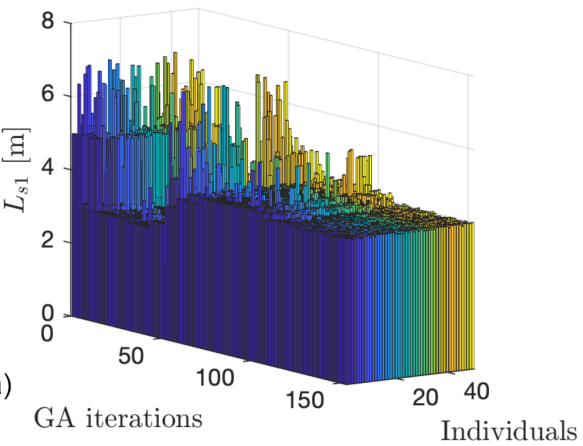

(c)
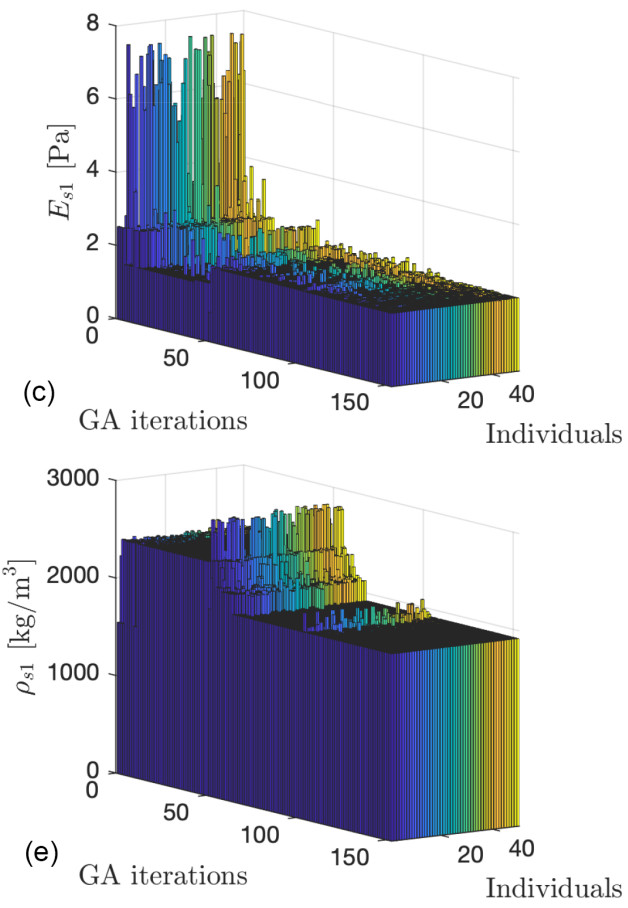
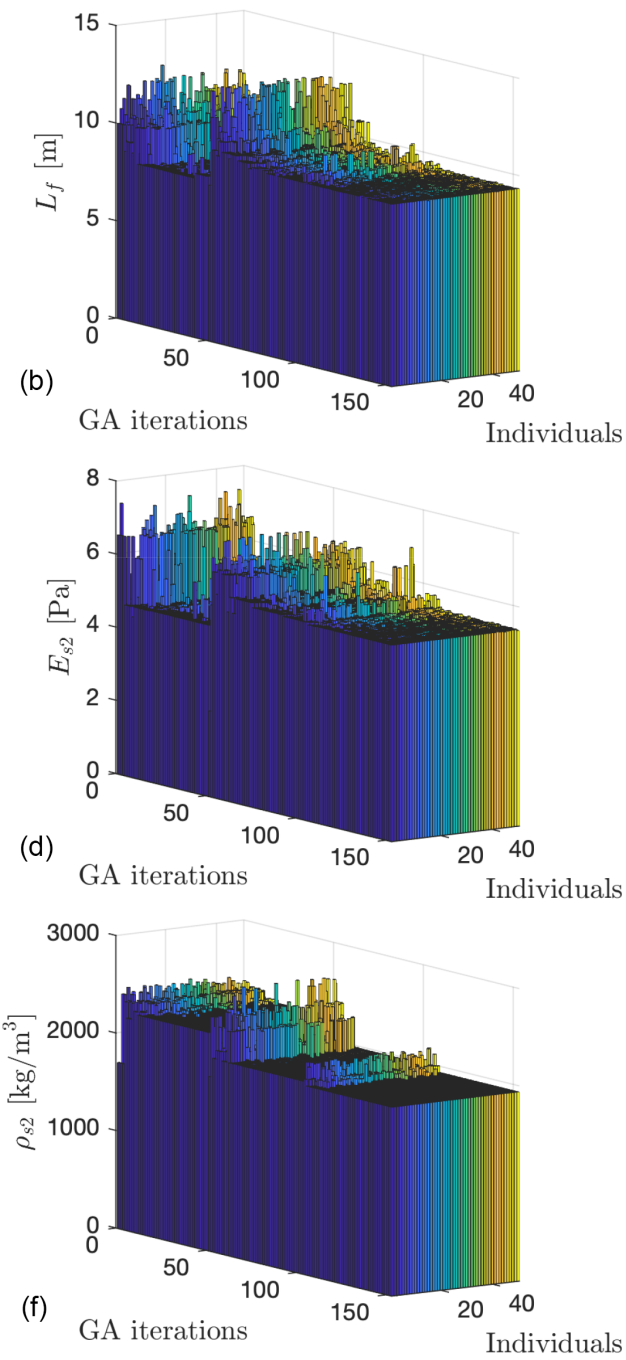

Figure 10: The histograms of (a) $L_{s 1}$, (b) $L_{f}$, (c) $E_{s 1}$, (d) $E_{s 2}$, (e) $\rho_{s 1}$, and (f) $\rho_{s 2}$ of the entire population of all the individuals at all the generations in Case 10 during the primary inversion. 
This Example 3 shows a proof of concept, potentially promising that $(i)$ we can detect fluid-filled cavities in a 2D or 3D domain by using the all-solid-element-based preliminary inversion of $E, G$, and $\rho$ and finding particular solid elements (of a high value of $E$, a low value of $G$, and the value of $\rho$, being similar to 1021 $\mathrm{kg} / \mathrm{m}^{3}$ ); then, $(\mathrm{ii})$ we can use a fluid-solid-element-based wave solver and identify the geometry of a fluidsolid interface and $E, G$, and $\rho$ of solid elements, surrounding fluid, in the 2D or 3D domain. The details of such 2D/3D extensions are shown below.

\section{Discussion}

We can extend this work into 2D and 3D settings in a manner such that one can identify the geometry of fluid-filled cavities and the material properties of surrounding soils by using an FWI algorithm (instead of $\mathrm{GA}$ ), which can be optionally combined with an artificial neural network (ANN). Although the GA can be a useful method to identify a small number of control parameters (e.g., up to 10) under a feasibility study like this presented research, the GA is considered to be a much less viable solution method than the FWI that is powered by the PDE-constrained optimization method when the number of control parameters is large in a case-such as one, where the material properties $E, G$, and $\rho$ of all the solid elements in a 2D or 3D setting are to be estimated. Namely, under the framework of FWI, we can employ the following two-step approach (i.e., preliminary to primary inversion) to (i) detect the existence of the fluid-filled voids and ( $i i$ ) accurately identify the geometry of the fluid-solid interface and the material proprieties of soils in 2D/3D settings.

- Preliminary inversion of FWI: First, one can approximately detect the existence of fluid-filled cavities in a 2D or 3D domain by using the all-solid-element-based inversion of $E, G$, and $\rho$ of solids. That is, in each iteration of this preliminary FWI, the forward solution is obtained for a guessed profiles of $E$, $G$, and $\rho$ in all the elements, which are solids, in a computational domain. Per each iteration, one can compute the value of a gradient of a misfit function-between measurement signal data at sensors and their computed counterparts due to a guessed profile-with respect to control parameters by using the solution of an adjoint problem, which utilizes the misfit at each sensor location as a driving force in an all-solid model. By using the gradient vector, the guessed profile can be updated, and this process is repeated until a termination condition is satisfied. Once one finds a particular area (i.e., an area of interest) of solid elements-with a high value of elasticity but a low value of shear modulus and a mass density that is similar to that of water, one can consider such an area as a fluid-filled cavity.

- Primary inversion of FWI: Subsequently, once this preliminary FWI is completed, we would switch the wave solver (for both forward and adjoint problems) such that fluid elements occupy the area of interest that was detected as a potential fluid-filled cavity from the preliminary inversion. In this primary FWI, one will update the following control parameters $-E, G$, and $\rho$ of the solid elements surrounding the fluid area, as well as the geometry parameters of the interface between fluid and solid elements. In that, we may have several possible methods of parameterizing the shape of the interface. Among them is using the Cartesian coordinates of the discretized nodes on the interface as control parameters. Our initial guessed profile of the control parameters of the interface will be based on the result from the preliminary all-solid FWI. For instance, Fig. 11 depicts a possible scenario of how one may approximate the initially-guessed geometry of the fluid-solid interface based on the result from the preliminary all-solid FWI. At each iteration of this primary FWI, one first solves the forward problem by using the guessed profile, then, solves the adjoint problem, and computes the gradient of a misfit function with respect to control parameters. When one computes the gradient with respect to the geometric control parameters, one can use the concept of a moving boundary linked with the total derivative (Jeong et al., 2009) that allows for the analytical evaluation of the gradient with respect to the shape parameters. By using the gradient, one updates the mesh such that it reflects the updated geometry parameters. Thus, this primary FWI should be linked, by virtue of the application programming interface (API), to a high-performance mesh generator (e.g., CUBIT) such that a new mesh is generated per each inversion iteration. Then, one repeats to calculate the forward and adjoint solutions and the gradient of a misfit for the new mesh and, then, updates the guessed profile of control parameters as well as the mesh until a termination condition is satisfied. The multiple frequency-continuation scheme can be employed under this primary FWI as well. As a forward and adjoint solver, one could potentially use a spectral element method (SEM) wave solver (Komatitsch and Tromp, 1999; Komatitsch et al., 2000, 2002; Komatitsch and Tromp, 2002; Tromp 
(a)

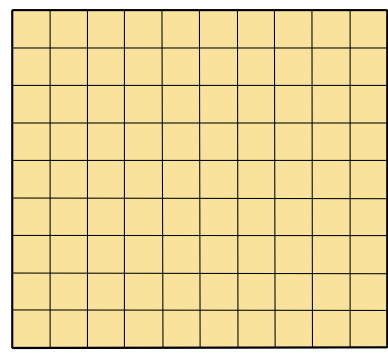

(c)

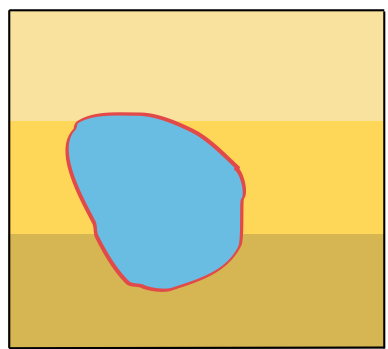

(b)

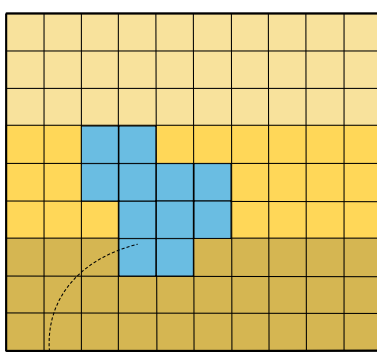

high $E$, low $G, \rho \approx 1021 \mathrm{~kg} / \mathrm{m}^{3}$

(d)

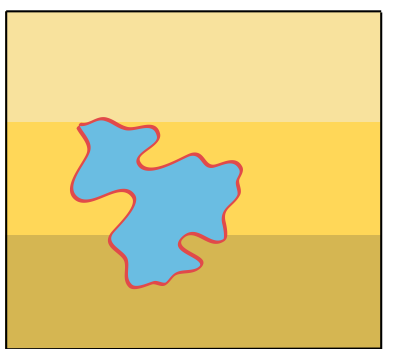

Figure 11: An exemplary sketch of our proposed method in 2D/3D settings: (a) an initial guess of a preliminary inversion, using a structured mesh, (b) the final result of the preliminary inversion, (c) an initial guess of a primary inversion, using an unstructured mesh, and (d) the final result of the primary inversion.

et al., 2008). It has already addressed the large computational cost in 2D/3D wave propagation analyses by virtue of a naturally-arisen diagonal mass matrix and explicit time integration, and the fluidsolid interface conditions and the absorbing boundary conditions are already implemented within the SEM solver (Lloyd et al., 2018). However, the moving boundary concept and the total derivativeassociated with updating the geometries of a fluid-solid interface-are not implemented in an SEM solver yet. Thus, the implementation of such new modeling in an SEM solver will enable the proposed primary FWI in 3D to be used for generating very accurate geophysical characterization data in Karst areas.

Alternatively, the 2D/3D extension can be investigated by using the combination of an artificial neural network (ANN) and FWI (henceforth, ANN2FWI) in the following two-step approach.

- Preliminary inversion of ANN2FWI: ANN can be used as an inversion solver in the preliminary inversion of ANN2FWI. We note that the considered inverse problem will be solved by using ANN as the combination of classification and regression problems not as a typical regression problem. The input-layer feature of the training data would be the measurement signals at sensor locations while the output-layer feature would be the classification of the possible type of elements (i.e., solid versus fluid) in a computational domain and the material properties ( $E, G$, and $\rho$ ) of solid elements (if a given element is classified as a solid element). By using the data, an ANN will be trained such that it can classify whether each element is a solid or fluid, and, then, once it is classified as a solid, the ANN will estimate its material properties. The details on the training of ANN are omitted in this paper because it is quite extensive and beyond the scope of this paper.

- Primary inversion of ANN2FWI: Once the preliminary inversion is completed, one can fine-tune its result by using the aforementioned primary FWI.

Our proposed method described above can be used as both initial screening (i.e., a preliminary inversion using all solid elements) and in-depth cavity characterization (i.e., a primary inversion using fluid-solid elements). We also note that initial screening may be done by using other geophysical methods, such as GPR and electrical resistivity. Furthermore, engineers or geologists can somehow know the existence of waterfilled underground cavities or internal soil erosion activities because of surface signs (e.g., depression or subsidence) or geological/hydrogeological phenomena (e.g., sinkhole raveling activities) in most of practical geoengineering application sites. Thus, engineers or geologists may have a combination of preliminary data from several different methods, including our proposed preliminary inversion, that will greatly benefit our proposed primary inversion using the fluid-solid elements in most cases.

It is critical, in the geo-engineering analysis and design, to know the dimension and location of an un- 
derground cavity. Thus, obtaining the accurate locations and shapes of fluid-filled voids and the material properties of surrounding soils from our proposed method will be instrumental to geotechnical engineers who perform further stability analysis by using the result from our proposed method.

\section{Summary}

We present a new multi-level GA-based inversion method combined with a frequency-continuation scheme. Three levels of GA are used, and, in each level, a pulse signal with a different dominant frequency is utilized to tackle the solution multiplicity of the presented inverse problem. Numerical results show the following findings about the proposed inversion method. First, it is feasible to estimate the depths of solid-fluid interfaces and Young's moduli of the surrounding solid layers by employing an acoustic-elastodynamic wave model and a measured dynamic response at a sensor on the top surface. Second, combining the multilevel GA-based optimizer with a frequency-continuation scheme improves the performance of identifying targeted control parameters. Third, the frequency-continuation scheme where the frequency is progressively decreased for each GA level is as effective as the conventional frequency-continuation scheme (i.e., increasing the frequency for each level) in the presented 1D setting. Lastly, a preliminary inversion using a solid-only model can address the uncertain presence of a fluid layer in a domain by uncovering that one of the layers has a very large value of Young's modulus (with a similar value to that of the bulk modulus of water) and a mass density being similar to that of water. After the preliminary inversion, a primary inversion-based on multiple frequency-level GA using a fluid-solid model-can adjust the fluid layer's depth information and the material properties of the soil layers. We also discussed how to extend the presented method in realistic 2D/3D settings. Such an extension may effectively detect water-filled underground cavities and also estimate the risk of potential urban cave-in and Karst sinkholes. Please also note that, in typical engineering projects, we do not rely on a single geophysical method no matter how advanced its theories or computation capacities are. Thus, the presented method and its extension can be an important supplement to existing testing methods rather than a stand-alone solution in practice.

\section{Data Availability}

Some or all data, models, or code generated or used during the study are available from the corresponding author by request.

- MATLAB code (.m format) of the presented inverse modeling that contains the optimization solver and the forward and adjoint wave solvers.

- MATLAB datasets (.mat format) of the presented numerical results.

\section{Acknowledgment}

This material is based upon work supported by the National Science Foundation under Award CMMI1855406 and CMMI-2044887. Any opinions, findings, and conclusions or recommendations expressed in this material are those of the authors and do not necessarily reflect the views of the National Science Foundation. The authors are also grateful for the support by the Faculty Research and Creative Endeavors (FRCE) Research Grant at Central Michigan University. 


\section{References}

Beck, B. F. (1986). A generalized genetic framework for the development of sinkholes and karst in Florida, U.S.A. Environmental Geology and Water Sciences, 8(1):5, ISBN: 1432-0495, DOI: 10.1007/BF02525554, https://doi.org/10.1007/BF02525554.

Bishop, I., Styles, P., Emsley, S., and Ferguson, N. (1997). The detection of cavities using the microgravity technique: case histories from mining and karstic environments. Geological Society, London, Engineering Geology Special Publications, 12(1):153-166.

Bloomberg, D., Upchurch, S., Hayden, M., and Williams, R. (1988). Cone-penetrometer exploration of sinkholes: Stratigraphy and soil properties. Environmental Geology and Water Sciences, 12(2):99-105.

Brown, L. T., Boore, D. M., and Stokoe, K. H. (2002). Comparison of shear-wave slowness profiles at 10 strong-motion sites from noninvasive sasw measurements and measurements made in boreholes. Bulletin of the Seismological Society of America, 92(8):3116-3133.

Bunks, C., Saleck, F. M., Zaleski, S., and Chavent, G. (1995). Multiscale seismic waveform inversion. Geophysics, 60(5):1457-1473.

Butler, D. K. (1984). Microgravimetric and gravity gradient techniques for detection of subsurface cavities. Geophysics, 49(7):1084-1096.

Cardarelli, E., Marrone, C., and Orlando, L. (2003). Evaluation of tunnel stability using integrated geophysical methods. Journal of Applied Geophysics, 52(2-3):93-102.

Coşkun, N. (2012). The effectiveness of electrical resistivity imaging in sinkhole investigations. International Journal of Physical Sciences, 7(15).

Fathi, A. (2015). Full-waveform inversion in three-dimensional PML-truncated elastic media: theory, computations, and field experiments. PhD thesis, The University of Texas at Austin.

Fathi, A., Kallivokas, L. F., and Poursartip, B. (2015a). Full-waveform inversion in three-dimensional PMLtruncated elastic media. Computer Methods in Applied Mechanics and Engineering, 296:39-72.

Fathi, A., Poursartip, B., and Kallivokas, L. F. (2015b). Time-domain hybrid formulations for wave simulations in three-dimensional PML-truncated heterogeneous media. International Journal for Numerical Methods in Engineering, 101(3):165-198.

Fathi, A., Poursartip, B., Stokoe II, K. H., and Kallivokas, L. F. (2016). Three-dimensional P-and S-wave velocity profiling of geotechnical sites using full-waveform inversion driven by field data. Soil Dynamics and Earthquake Engineering, 87:63-81.

Fehdi, C., Nouioua, I., Belfar, D., Djabri, L., and Salameh, E. (2014). Detection of underground cavities by combining electrical resistivity imaging and ground penetrating radar surveys: A case study from draa douamis area (north east of algeria). In H2Karst Research in Limestone Hydrogeology, pages 69-82. Springer.

Guzina, B. B., Fata, S. N., and Bonnet, M. (2003). On the stress-wave imaging of cavities in a semi-infinite solid. International Journal of Solids and Structures, 40(6):1505-1523.

Jeong, C. and Kallivokas, L. F. (2008). Inverse scatterer reconstruction in a halfplane using surficial SH line sources. CMES: Computer Modeling in Engineering \& Sciences, 35(1):49-72.

Jeong, C., Na, S.-W., and Kallivokas, L. F. (2009). Near-surface localization and shape identification of a scatterer embedded in a halfplane using scalar waves. Journal of Computational Acoustics, 17(03):277308, http://www.worldscientific.com/doi/abs/10.1142/S0218396X09003963.

Jeong, C., Peixoto, A. C. S., Aquino, A., Lloyd, S., and Arhin, S. (2017). Genetic algorithm-based acousticsource inversion approach to detect multiple moving wave sources of an arbitrary number. Journal of Computing in Civil Engineering, 31(5):04017020.

Jung, J., Jeong, C., and Taciroglu, E. (2013). Identification of a scatterer embedded in elastic heterogeneous media using dynamic XFEM. Computer Methods in Applied Mechanics and Engineering, 259:50-63.

Jung, J. and Taciroglu, E. (2014). Modeling and identification of an arbitrarily shaped scatterer using dynamic XFEM with cubic splines. Computer Methods in Applied Mechanics and Engineering, 278:101-118, http: //www.sciencedirect.com/science/article/pii/S0045782514001467. 
Jung, J. and Taciroglu, E. (2016). A divide-alternate-and-conquer approach for localization and shape identification of multiple scatters in heterogeneous media using dynamic XFEM. Inverse Problems and Imaging, 10(1).

Kallivokas, L., Fathi, A., Kucukcoban, S., Stokoe, K., Bielak, J., and Ghattas, O. (2013). Site characterization using full waveform inversion. Soil Dynamics and Earthquake Engineering, 47:62-82.

Kang, J. W. and Kallivokas, L. F. (2010a). The inverse medium problem in 1D pml-truncated heterogeneous semi-infinite domains. Inverse Problems in Science and Engineering, 18(6):759-786, DOI: 10.1080/17415977.2010.492510.

Kang, J. W. and Kallivokas, L. F. (2010b). Mixed unsplit-field perfectly matched layers for transient simulations of scalar waves in heterogeneous domains. Computational Geosciences, 14(4):623-648.

Kang, J. W. and Kallivokas, L. F. (2011). The inverse medium problem in heterogeneous PML-truncated domains using scalar probing waves. Computer Methods in Applied Mechanics and Engineering, 200(1):265-283, http: //www. sciencedirect.com/science/article/pii/S0045782510002434.

Kiflu, H., Kruse, S., Loke, M., Wilkinson, P., and Harro, D. (2016). Improving resistivity survey resolution at sites with limited spatial extent using buried electrode arrays. Journal of Applied Geophysics, 135:338355.

Kim, Y. J., Nam, B. H., Shamet, R., Soliman, M., and Youn, H. (2020). Development of sinkhole susceptibility map of east central florida. Natural Hazards Review, 21(4):04020035, DOI: 10.1061/(ASCE) NH.1527-6996.0000404.

Komatitsch, D., Barnes, C., and Tromp, J. (2000). Wave propagation near a fluid-solid interface: A spectralelement approach. Geophysics, 65(2):623-631.

Komatitsch, D., Ritsema, J., and Tromp, J. (2002). The spectral-element method, Beowulf computing, and global seismology. Science, 298(5599):1737-1742.

Komatitsch, D. and Tromp, J. (1999). Introduction to the spectral element method for three-dimensional seismic wave propagation. Geophysical Journal International, 139:806-822.

Komatitsch, D. and Tromp, J. (2002). Spectral-element simulations of global seismic wave propagation - I. Validation. Geophysical Journal International, 149:390-412.

Kucukcoban, S., Goh, H., and Kallivokas, L. F. (2019). On the full-waveform inversion of lame parameters in semi-infinite solids in plane strain. International Journal of Solids and Structures, 164:104 - 119, ISSN: 0020-7683, DOI: https://doi.org/10.1016/j.ijsolstr.2019.01.019.

Kucukcoban, S. and Kallivokas, L. F. (2011). Mixed perfectly-matched-layers for direct transient analysis in 2D elastic heterogeneous media. Computer Methods in Applied Mechanics and Engineering, 200:57-76.

Kucukcoban, S. and Kallivokas, L. F. (2013). A symmetric hybrid formulation for transient wave simulations in PML-truncated heterogeneous media. Wave Motion, 50:57 - 79.

Lloyd, S., Jeong, C., Nath Gharti, H., Vignola, J., Judge, J., and Tromp, J. (2018). Spectral-element simulations of acoustic waves induced by a moving underwater source. Journal of Theoretical and Computational Acoustics.

Lloyd, S. F., Jeong, C., and Okutsu, M. (2016). Numerical investigation on the feasibility of estimating the thickness of europa\&\#x2019;s ice shell by a planned impact. Journal of Aerospace Engineering, 29(5):04016035, https : //ascelibrary . org/doi/abs/10.1061/\%28ASCE\%29AS. 1943-5525. 0000621.

Mashayekh, H., Kallivokas, L. F., and Tassoulas, J. L. (2018). Parameter estimation in layered media using dispersion-constrained inversion. Journal of Engineering Mechanics, 144(11):04018099, DOI: 10.1061/ (ASCE) EM.1943-7889.0001527.

Mirzanejad, M., Tran, K. T., McVay, M., Horhota, D., and Wasman, S. J. (2020). Sinkhole detection with 3d full seismic waveform tomography. GEOPHYSICS, 85(5):B169-B179, DOI: 10.1190/geo2019-0490.1, https://doi.org/10.1190/geo2019-0490.1. 
Mochales, T., Casas, A., Pueyo, E., Pueyo, O., Román, M., Pocoví, A., Soriano, M., and Ansón, D. (2008). Detection of underground cavities by combining gravity, magnetic and ground penetrating radar surveys: a case study from the zaragoza area, ne spain. Environmental Geology, 53(5):1067-1077.

$\mathrm{Na}$, S. and Kallivokas, L. (2008). Continuation schemes for shape detection in inverse acoustic scattering problems. Computer Modeling in Engineering \& Sciences, 35(1):73-90.

Nam, B. H., Kim, Y. J., and Youn, H. (2020). Identification and quantitative analysis of sinkhole contributing factors in Florida's Karst. Engineering Geology, 271:105610, ISSN: 0013-7952, https: //www . sciencedirect.com/science/article/pii/S0013795219317703.

Nam, B. H. and Shamet, R. (2020). A preliminary sinkhole raveling chart. Engineering Geology, 268:105513, ISSN: 0013-7952, https://www.sciencedirect.com/science/article/pii/ S0013795219312669.

Nam, B. H., Shamet, R., Soliman, M., Wang, D., and Yun, H.-B. (2018). Development of a sinkhole risk evaluation program.

Newmark, N. M. (1959). A method of computation for structural dynamics. In Proc. ASCE, volume 85, pages 67-94.

Pakravan, A., Kang, J. W., and Newtson, C. M. (2016). A Gauss-Newton full-waveform inversion for material profile reconstruction in viscoelastic semi-infinite solid media. Inverse Problems in Science and Engineering, 24(3):393-421, ISSN: 1741-5977, DOI: 10.1080/17415977.2015.1046861, http: //dx.doi.org/10.1080/17415977.2015.1046861.

Petryk, H. and Mroz, Z. (1986). Time derivatives of integrals and functionals defined on varying volume and surface domains. Archives of Mechanics, ISSN: 0373-2029.

Poul, M. K. and Zerva, A. (2018). Time-domain PML formulation for modeling viscoelastic waves with Rayleigh-type damping in an unbounded domain: Theory and application in ABAQUS. Finite Elements in Analysis and Design, 152:1 - 16, ISSN: 0168-874X, http://www.sciencedirect.com/science/ article/pii/S0168874X18300313.

Roesset, J. M., Chang, D.-W., Stokoe, I., Kenneth, H., and Aouad, M. (1990). Modulus and thickness of the pavement surface layer from sasw tests. Transportation Research Record, (1260).

Shamet, R. M., Perez, A., and Nam, B. H. (2017). Sinkhole risk evaluation: Detection of raveled soils in Central Florida's Karst geology using CPT. In Geo-Risk 2017, pages 257-266.

Tihansky, A. B. (1999). Sinkholes, west-central florida. Land subsidence in the United States: US geological survey circular, 1182:121-140.

Tran, K. T. and McVay, M. (2012). Site characterization using Gauss-Newton inversion of 2-D full seismic waveform in the time domain. Soil Dynamics and Earthquake Engineering, 43:16-24, ISSN: 02677261, DOI: 10.1016/j.soildyn.2012.07.004, https://linkinghub.elsevier.com/retrieve/pii/ S0267726112001613.

Tran, K. T., McVay, M., Faraone, M., and Horhota, D. (2013). Sinkhole detection using 2d full seismic waveform tomography. GEOPHYSICS, 78(5):R175-R183, DOI: 10.1190/geo2013-0063.1, https : //doi.org/10.1190/geo2013-0063.1.

Tromp, J., Komattisch, D., and Liu, Q. (2008). Spectral-element and adjoint methods in seismology. Communications in Computational Physics, 3(1):1-32.

Van Schoor, M. (2002). Detection of sinkholes using 2D electrical resistivity imaging. Journal of Applied Geophysics, 50(4):393-399.

Xiao, H., Kim, Y. J., Nam, B. H., and Wang, D. (2016). Investigation of the impacts of local-scale hydrogeologic conditions on sinkhole occurrence in east-central florida, usa. Environmental Earth Sciences, 75(18):1274, ISBN: 1866-6299, DOI: 10.1007/s12665-016-6086-3, https://doi.org/10.1007/ s12665-016-6086-3. 